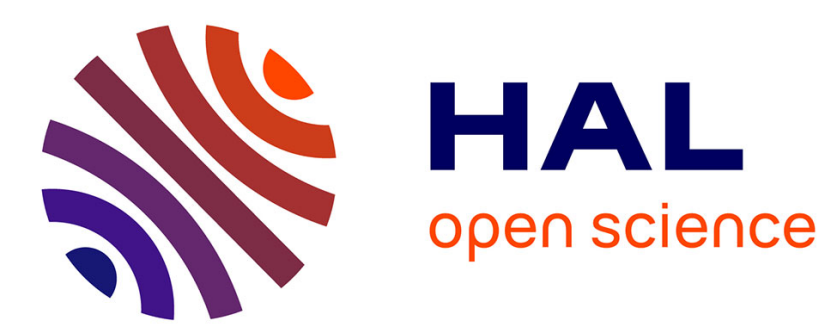

\title{
Nonlinear adaptive output feedback control of series resonant DC-DC converters
}

\author{
Fouad Giri, O. El Maguiri, H. El Fadil, Fatima Zara Chaoui
}

\section{To cite this version:}

Fouad Giri, O. El Maguiri, H. El Fadil, Fatima Zara Chaoui. Nonlinear adaptive output feedback control of series resonant DC-DC converters. Control Engineering Practice, 2011, 19 (10), pp.Pages 1238-1251. 10.1016/j.conengprac.2011.07.012 . hal-01060434

\section{HAL Id: hal-01060434 https://hal.science/hal-01060434}

Submitted on 9 Sep 2014

HAL is a multi-disciplinary open access archive for the deposit and dissemination of scientific research documents, whether they are published or not. The documents may come from teaching and research institutions in France or abroad, or from public or private research centers.
L'archive ouverte pluridisciplinaire HAL, est destinée au dépôt et à la diffusion de documents scientifiques de niveau recherche, publiés ou non, émanant des établissements d'enseignement et de recherche français ou étrangers, des laboratoires publics ou privés. 


\title{
Nonlinear adaptive output feedback control of series resonant DC-DC converters
}

\author{
F. Giri*, O. El Maguiri, H. El Fadil, F.Z. Chaoui \\ GREYC lab, UMR CNRS, University of Caen Basse-Normandie, Caen, France
}

\begin{abstract}
A B S T R A C T
The problem of regulating the output voltage of DC-to-DC series resonant converters (SRC) is addressed. The difficulty is threefold: (i) the converter model involves discontinuous and highly nonlinear terms and is controlled through a modulating frequency signal; (ii) all state variables are not accessible to measurements; (iii) the load is uncertain and may even be varying. An adaptive output feedback controller, involving online state variable estimation, is designed and shown to ensure quite satisfactory tracking performances. The controller development is performed using the adaptive backstepping control approach combined with the high-gain observer design technique.
\end{abstract}

\section{Introduction}

Series and parallel resonant DC-to-DC converters have been given a great deal of interest in the power electronic literature. Compared to (hard) switched converters, resonant converters present several advantages e.g. they provide much higher power supplies. Indeed, the absence of switched components considerably reduces power losses and improves the conversion efficiency of resonant converters. Furthermore, since the losses (in hard switched converters) are proportional to the switching frequency, it turns out that resonant converters can operate at much higher switching frequencies than comparable PWM converters (Erickson \& Maksimivic, 2001; Kazimierczuk \& Czarkowski, 2011). On the other hand, it is widely recognized (e.g. Rashid, 2001, p. 272) that resonant converters are also useful for not necessarily high power applications when weight and size reduction is sought. This feature is particularly useful in embedded applications. The point is that resonant converters are more complex to control due to their highly nonlinear dynamics. Furthermore, being supplied by bipolar square signal generators, they only can be controlled by unusual input signals e.g. switching frequency, current/voltage phase shifting in the inverter output or phase shifting between the inverter and rectifier control signal. These considerations make SRC modeling a particularly hard task. In this respect, approximate smooth state space models have been proposed using generalized averaging procedures (Sun \& Grotstohen, 1993), harmonic approximation and Fourier transformations (Wong \& Brown, 1995). Small signal models for series and parallel resonant converters have been obtained in (Vorperian, 1989).

\footnotetext{
* Corresponding author.

E-mail address: fouadgiri@yahoo.fr (F. Giri).
}

In addition to model complexity, the difficulty of the control problem (for resonant power converters) also lies in the fact that the state variables of these circuits are not all accessible to measurements. Finally, the converter load, generally modeled by a resistance, is usually uncertain and/or varying. These difficulties make output feedback control of resonant power converters a challenging problem.

In the present work, the focus is made on the problem of controlling the DC-to-DC SRC depicted by Fig. 1. This is representative of a wide class of circuits considered in the control literature. Following the generalized averaging procedure (Sun \& Grotstohen, 1993), a fifth order state-space model is developed for the considered circuit. The switching frequency stands up as the system control signal. From the control design viewpoint, the difficulty is threefold: (i) system nonlinearity and discontinuity; (ii) some state variables are not accessible to measurements; (iii) the control signal comes in explicitly in all state equations. In recent years, the control problem has been coped with following various control strategies including hybrid flatness control (SiraRamirez \& Silva-Ortega, 2002), 'resonant tanks variables' optimal control (Oruganti, Young, \& Lee, 2002), sliding mode control (Sosa, Castilla, De Vicuna, Miret, \& Cruz, 2005) and passivity control (Carasco, Escobar, \& Ortega, 2000). In these works, the converter load was generally supposed to be known and the state variables measurable. As already pointed out, these assumptions are generally not fulfilled in practice i.e. the load is generally uncertain (ill-known and/or varying) and all state variables are not accessible to measurements. Presently, a new control strategy is developed without resorting to these assumptions. Following (Giri, Liu, El Fadil, \& Elmaguiri, 2009), a high gain observer is first designed to get estimates of the unavailable state variables. Based on the resulting observed model, an adaptive output control law 
is designed, using the tuning-functions backstepping technique (Krstic, Kanellakopoulos, \& Kokotovic, 1995). Adaptation concerns the unknown converter load. It is worth recalling that, unlike linear systems, the separation principle does not systematically apply to nonlinear systems (Atassi \& Khalil, 2000). Presently, it happens that the unknown load only enters the equation of the output voltage (which is measurable). This fact will prove to be crucial in obtaining a stabilizing output feedback controller by direct combination of the observer and the adaptive control law. Furthermore, a parameter projection will be introduced in the parameter adaptive law (that online estimates the load) to prevent possible parameter estimate drift (that otherwise can result from the presence of state estimation errors in the observed model). The output adaptive controller thus obtained is formally shown to achieve quite interesting performances. Specifically, the output reference tracking error vanishes asymptotically with a specified convergence rate. This theoretical performance is confirmed by several simulations which also prove the good robustness of the developed controller to modeling errors.

The paper is organized as follows: the series resonant converter under study is described and modeled in Section 2; a state observer is designed and analyzed in Section 3; the adaptive output feedback controller is designed and analyzed in Section 4; the controller performances are illustrated by simulation in Section 5 .

\section{Series resonant converter modeling}

Resonant converters contain resonant LC networks whose voltage and current waveforms vary sinusoidally during one or more subintervals of each switching period. Depending on how the resonant networks are combined with other circuit configurations, one can obtain several types of resonant converters. Fig. 1 shows a general functional diagram of resonant converters and Fig. 2 shows the class of series resonant DC-to-DC full bridge converters under study. There, the transformer is resorted to potential separation and both the transformer and inductance $(L)$ are expected to operate at relatively large frequencies and powers. Ferrite cores are most suitable for this purpose.

\subsection{SRC physical modeling}

Applying the usual electrical laws to the series resonant DC-toDC converter of Fig. 2 one gets the following state-space repre- sentation of the system:

$L \frac{d i}{d t}=-v-\frac{v_{o}}{n} \operatorname{sgn}(i)+v_{i}$

$C \frac{d v}{d t}=i$

$C_{0} \frac{d v_{0}}{d t}=\frac{a b s(i)}{n}-\frac{v_{o}}{R}$

where $v$ and $i$ denote the resonant tank voltage and current respectively; $v_{o}$ is the output voltage supplying the load (here represented by a resistance $R$ ); $v_{i}$ is the inverter output voltage; $L$ and $C$ designate, respectively, the inductance and capacitance of the resonant tank; and $n$ is the amplification ratio of the HF-transformer, the resonant frequency is denoted $f_{0}=1 / 2 \pi \sqrt{L C}$.

The voltage $v_{i}$ is a periodic square wave of variable amplitude and frequency. Usually, the amplitude is maintained constant, leaving the frequency as the only possible control variable. In the rest of the paper, this voltage is assumed to be of the form $v_{i}=E \operatorname{sgn}(\sin (\omega t))$ with $E$ a real constant representing the source amplitude. The varying switching-frequency $\omega(\mathrm{rd} / \mathrm{s})$ turns out to be the control input signal. For the sake of simplicity, no parasitic effects are considered, at this stage, in switches, inductance and capacitance.

\subsection{First-harmonic approximation model}

From the control design viewpoint, the model (1a)-(1c) presents a major drawback, namely the control signal $\omega$ comes in nonlinearly. Following (Sun \& Grotstohen, 1993), a controloriented simpler version of (1a)-(1c) will now be developed applying the first harmonic approximation. This approach relies on the assumption that the solution of a nonlinear oscillator system can be expanded as a Fourier series with time-varying coefficients. Specifically, one has the standard expressions

$$
\begin{aligned}
& x(t, s) \stackrel{\text { def }}{=} x(t-T+s)=\sum_{k=-\infty}^{+\infty}\left\langle x_{k}\right\rangle(t) e^{j k \omega(t-T+s)}, \quad s \in[0 T] \\
& \left\langle x_{k}\right\rangle(t)=\frac{1}{T} \int_{0}^{T} x(t-T+s) e^{-j k \omega(t-T+s)} d s
\end{aligned}
$$

with $\omega=2 \pi / T$. The coefficients $\left\langle x_{k}\right\rangle$ undergo the following equation:

$$
\frac{d\left\langle x_{k}\right\rangle}{d t}=\left\langle\frac{d}{d t} x\right\rangle_{k}(t)-j k \omega\left\langle x_{k}\right\rangle(t)
$$

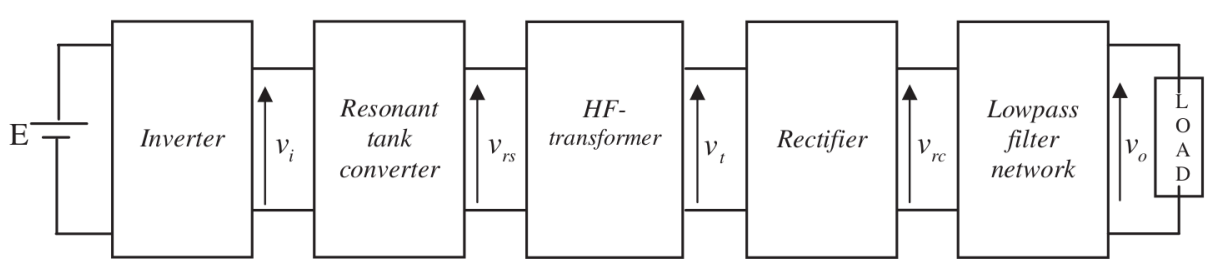

Fig. 1. Functional diagram of DC resonant converters.

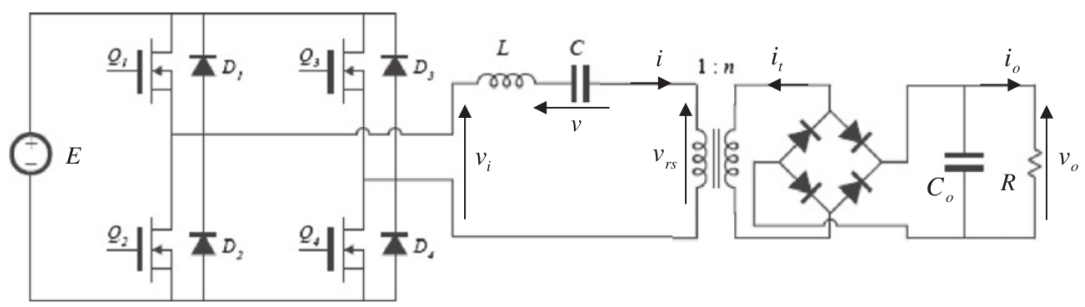

Fig. 2. Series resonant converter under study. 
In the case $x(t)$ is generated by a nonlinear system $\dot{x}=f(x, u)$ where $u$ denotes the control signal, it follows from (4) that:

$\frac{d\left\langle x_{k}\right\rangle}{d t}=\langle f(x, u)\rangle_{k}(t)-j k \omega\left\langle x_{k}\right\rangle(t)$

The first-harmonic-approximation based modeling relies on the following standard assumptions (e.g. Carasco et al., 2000):

A1. The voltage $v$ and current $i$ are well approximated by their (time-varying) first harmonics (denoted $V_{1}$ and $I_{1} e^{j \psi}$, respectively).

A2. The time scale of the output filter is much larger than the resonant tank so that the ripple appearing in the output voltage can be neglected and $v_{o}$ can be well approximated by its DC-component. i.e. $v_{o}=V_{o}$ where $V_{o}$ is the DC-component of the (time varying) Fourier series of $v_{o}$.

A3. All physical variables (i.e. $V_{o}, V_{1}, I_{1}$ ) are bounded. This is not an issue because all real-life physical systems involve bounded signals.

Applying (5) with $k=1$ to equations (1a)-(1c), one obtains the following 'first-harmonic' nonlinear model of the SRC (Sun \& Grotstohen, 1993):

$\frac{d I_{1}}{d t}=-j \omega I_{1}+\frac{1}{L}\left[-V_{1}-\frac{2}{n \pi} V_{0} e^{j \Psi}-j \frac{2 E}{\pi}\right]$

$\frac{d V_{1}}{d t}=-j \omega V_{1}+\frac{1}{C} I_{1}$

$\frac{d V_{0}}{d t}=\frac{4}{n \pi C_{0}} a b s\left(I_{1}\right)-\frac{V_{o}}{R C_{0}}$

The 'harmonic' model (6)-(8) is quite representative of the SRC due to Assumptions 1 and 2. The benefit of this model compared to (1a)-(1c) is that the control signal $\omega$ comes in linearly. However, it still is not suitable for control/observer designs because it involves complex variables and parameters. To get a convenient state-space model (with real state variables), introduce the following notations:

$I_{1}=x_{1}+j x_{2}, \quad V_{1}=x_{3}+j x_{4}, \quad V_{o}=x_{5}$

Substituting (9) in (6)-(8) yields the following state-space representation:

$\dot{x}_{1}=x_{2} u-\frac{x_{3}}{L}-\frac{2 x_{5}}{n \pi L} \frac{x_{1}}{\sqrt{x_{1}^{2}+x_{2}^{2}}}$

$\dot{x}_{2}=-x_{1} u-\frac{x_{4}}{L}-\frac{2 x_{5}}{n \pi L} \frac{x_{2}}{\sqrt{x_{1}^{2}+x_{2}^{2}}}-\frac{2 E}{\pi L}$

$\dot{x}_{3}=x_{4} u+\frac{x_{1}}{C}$

$\dot{x}_{4}=-x_{3} u+\frac{x_{2}}{C}$

$\dot{x}_{5}=\frac{4}{n \pi C_{0}} \sqrt{x_{1}^{2}+x_{2}^{2}}-\frac{x_{5}}{C_{o}} \theta$

where $u \stackrel{\text { def }}{=} \omega$ and $\theta=1 / R$. The only quantities that are accessible to measurements are

$x_{5}=V_{0}, \quad \sqrt{x_{1}^{2}+x_{2}^{2}}=I_{1}, \quad \sqrt{x_{3}^{2}+x_{4}^{2}}=V_{1}$

Remark 1. It is worth noting that the amplitudes of the resonant tank (fundamental) current and voltage stay bounded away from zero i.e. $\min \left(\left|I_{1}\right|,\left|V_{1}\right|\right) \geq \varepsilon>0$ for some threshold $\varepsilon$. Indeed, it readily follows from Assumption A2 that the voltage $v_{r s}(t)$, at the HF-transformer input, is a square wave. Furthermore, the fundamental component of $v_{r s}(t)$ is in phase with the resonant tank output current $i$, due to Assumption A1. Therefore, the rectifier behaves as a resistance load vis-à-vis to the resonant tank network (Fig. 1). It can be easily checked that the load equivalent resistance has the following value:

$R_{e}=\frac{8}{(n \pi)^{2}} R$

Accordingly, the input impedance of the resonant tank network has the following magnitude:

$\left|Z_{\text {in }}\right|=\sqrt{R_{e}^{2}+\left(2 \pi f L-\frac{1}{2 \pi f C}\right)^{2}}$

Due to semiconductor device characteristics, the switching frequency $f$ is physically limited to some finite value, i.e. $0<f<f_{\max }<\infty$. Then, it follows that the resonant current and voltage magnitudes $I_{1}$ and $V_{1}$ stay away from some nonzero thresholds $I_{1 \mathrm{~min}}$ and $V_{1 \mathrm{~min}}$, respectively, with:

$$
\begin{aligned}
I_{1 \min } & =\frac{\frac{4 E}{\pi}}{\max _{0<f<f_{\max }\left|Z_{\text {in }}\right|}}=\frac{4 E}{\pi \sqrt{R_{e}^{2}+\left(2 \pi f_{\max } L-\frac{1}{2 \pi f_{\max } C}\right)^{2}}} \\
V_{1 \min } & =\frac{1}{2 \pi^{2} f_{\max } C} \frac{4 E}{\sqrt{R_{e}^{2}+\left(2 \pi f_{\max } L-\frac{1}{2 \pi f_{\max } C}\right)^{2}}}
\end{aligned}
$$

That is, $\min \left(\left|I_{1}\right|,\left|V_{1}\right|\right) \geq \varepsilon>0$ with $\varepsilon=\min \left(I_{1 \mathrm{~min}}, V_{1 \mathrm{~min}}\right)$.

\section{Observer design and analysis}

The model (10a)-(10e) involves state variables, namely $\left(x_{1}, \ldots, x_{4}\right)$, that are not accessible to measurements. Therefore, an observer has to be designed so that accurate estimates of the unmeasured variables can be obtained online. State observers for nonlinear systems can be obtained following different design approaches (see e.g. Besançon, 2007). A particular attention has been paid to the high-gain technique due its ability to quickly reconstruct the system states and reject modeling disturbances (e.g. Ahrens \& Khalil, 2009). Furthermore, the high-gain observer is featured by its mathematical simplicity as it involves no (online) resolution of any differential equation. The expression of this observer is immediately obtained when the system fits a canonical model form composed of a fixed linear dynamic subsystem and a triangular controlled subsystem. The development of this form for the model (10a)-(10e) is coped with in Section 3.1.

\subsection{Model transformation}

Let us introduce the following diffeomorphism map:

$$
\begin{aligned}
\Psi: I R^{5} & \rightarrow I R^{7} \\
& x \rightarrow Z=\Psi(x)
\end{aligned}
$$

with

$$
x=\left(\begin{array}{l}
x_{1} \\
x_{2} \\
x_{3} \\
x_{4} \\
x_{5}
\end{array}\right) ; \quad z=\Psi(x)=\left(\begin{array}{c}
z_{1} \\
z_{2} \\
z_{3} \\
z_{4} \\
z_{5} \\
z_{6} \\
z_{7}
\end{array}\right)=\left(\begin{array}{c}
\sqrt{x_{1}^{2}+x_{2}^{2}} \\
\sqrt{x_{3}^{2}+x_{4}^{2}} \\
x_{1} x_{3}+x_{2} x_{4} \\
x_{2} \\
x_{4} \\
x_{1} \\
x_{3}
\end{array}\right)
$$


Notice that, in the new coordinate system, the first two variables (i.e. $z_{1}$ and $z_{2}$ ) are accessible to measurement, due to (11). This suggests the introduction of the output vector $y=\left[\begin{array}{ll}z_{1} & z_{2}\end{array}\right]^{T}$. Using (13b), it follows from $(10 \mathrm{a})-(10 \mathrm{e})$ that the new state vector $z$ undergoes the following equations:

$\dot{z}_{1}=-\frac{z_{3}}{L z_{1}}-\frac{2 x_{5}}{n \pi L}-\frac{2 E z_{4}}{\pi L z_{1}}$

$\dot{z}_{2}=\frac{z_{3}}{z_{2} C}$

$\dot{z}_{3}=-\frac{z_{2}^{2}}{L}+\frac{z_{1}^{2}}{C}-\frac{2 z_{3} x_{5}}{n \pi L z_{1}}-\frac{2 E z_{5}}{\pi L}$

$\dot{z}_{4}=-z_{6} u-\frac{z_{5}}{L}-\frac{2 x_{5} z_{4}}{n \pi L z_{1}}-\frac{2 E}{\pi L}$

$\dot{z}_{5}=-z_{7} u+\frac{z_{4}}{C}$

$\dot{z}_{6}=z_{4} u-\frac{z_{7}}{L}-\frac{2 x_{5} z_{6}}{n \pi L z_{1}}$

$\dot{z}_{7}=z_{5} u+\frac{z_{6}}{C}$

$y=\left[\begin{array}{ll}z_{1} & z_{2}\end{array}\right]^{T}$

To give to (14a)-(14h) a compact form, let us introduce the following notations:

$z^{1}=\left[\begin{array}{ll}z_{1} & z_{2}\end{array}\right]^{T} ; \quad z^{2}=\left[\begin{array}{ll}z_{3} & z_{4}\end{array}\right]^{T} ; \quad z^{3}=\left[\begin{array}{ll}z_{5} & z_{6}\end{array}\right]^{T} ; \quad z^{4}=z_{7}$

$\bar{C}=\left[\begin{array}{llll}I_{2 \times 2} & 0_{2 \times 2} & 0_{2 \times 2} & 0_{2 \times 1}\end{array}\right]$

where $I_{2 \times 2}$ denotes the $2 \times 2$ identity matrix and $0_{i \times j}$ the $i \times j$ null matrix. It is readily seen that $z=\left[\begin{array}{llll}z^{1 T} & z^{2 T} & z^{3 T} & z^{4 T}\end{array}\right]^{T}$. Then, the system (14a)-(14g) can be given the following compact form:

$\left\{\begin{array}{l}\dot{z}=F(z, u) z+G(u, z) \\ y=z^{1}=\bar{C} z\end{array}\right.$

with

$F(z, u)=\left[\begin{array}{cccc}0 & F_{1}\left(z^{1}, u\right) & 0 & 0 \\ 0 & 0 & F_{2}\left(z^{1}, z^{2}, u\right) & 0 \\ 0 & 0 & 0 & F_{3}\left(z^{1}, z^{2}, z^{3}, u\right) \\ 0 & 0 & 0 & 0\end{array}\right]$

where the blocs $F_{k}(k=1,2,3)$ are defined by

$F_{1}\left(z^{1}, u\right)=\left(\begin{array}{cc}\frac{-1}{L z_{1}} & \frac{-2 E}{\pi L z_{1}} \\ \frac{1}{C z_{2}} & 0\end{array}\right)$

$F_{2}\left(z^{1}, z^{2}, u\right)=\left(\begin{array}{cc}\frac{-2 E}{\pi L} & 0 \\ \frac{-1}{L} & -u\end{array}\right)$

$F_{3}\left(z^{1}, z^{2}, z^{3}, u\right)=\left(\begin{array}{l}-u \\ -\frac{1}{L}\end{array}\right)$

$G(u, z)$ is a vector field of the form

$G(z, u)=\left[\begin{array}{c}G_{1}\left(z^{1}, u\right) \\ G_{2}\left(z^{1}, z^{2}, u\right) \\ G_{3}\left(z^{1}, z^{2}, z^{3}, u\right) \\ G_{4}(z, u)\end{array}\right]$ with

$G_{1}\left(z^{1}, u\right)=\left(\begin{array}{c}\frac{-2 x_{5}}{n \pi L} \\ 0\end{array}\right)$

$G_{2}\left(z^{1}, z^{2}, u\right)=\left(\begin{array}{c}-\frac{z_{2}^{2}}{L}+\frac{z_{1}^{2}}{C}-\frac{2 z_{3} x_{5}}{n \pi L z_{1}} \\ \frac{-2 x_{5} z_{4}}{n \pi L z_{1}}-\frac{2 E}{\pi L}\end{array}\right)$

$G_{3}\left(z^{1}, z^{2}, z^{3}, u\right)=\left(\begin{array}{c}\frac{z_{4}}{C} \\ z_{4} u-\frac{2 x_{5} z_{6}}{n \pi L z_{1}}\end{array}\right)$

$G_{4}(z, u)=\left(z_{5} u+\frac{z_{6}}{C}\right)$

The new system representation defined by (16) to (20d) fits well the high-gain canonical form (e.g. Besançon, 2007) and satisfies the required conditions i.e. $y=z^{1}$ is measurable and $\operatorname{dim} z^{1} \geq \operatorname{dim} z^{2} \geq \operatorname{dim} z^{3}$. This form will now be based upon to design the observer. As a first step, various auxiliary variables needed in the observer definition will be computed in Section 3.2. There, the following properties will prove to be useful:

Proposition 1. Consider the system (10a)-(10e), also represented by (14a)-(14e) or (16). Then, the matrices $F_{k}^{T}(u, z) F_{k}(u, z)(k=1,2,3)$ are bounded and positive definite. Furthermore, the functions $G(u, z)$, $F_{1}(u, z), F_{2}(u, z)$ and $F_{3}(u, z)$ are globally Lipschitz with respect to $z$ uniformly in $u$.

Proof. Let us prove the first part for $k=1$. From (18a) one has

$F_{1}^{T}(u, z) F_{1}(u, z)=\left(\begin{array}{cc}\frac{1}{\left(L z_{1}\right)^{2}}+\frac{1}{\left(C z_{2}\right)^{2}} & \frac{2 E}{\pi\left(L z_{1}\right)^{2}} \\ \frac{2 E}{\pi\left(L z_{1}\right)^{2}} & \left(\frac{2 E}{\pi L z_{1}}\right)^{2}\end{array}\right)$

From Remark 1 and definitions (11) and (13b), one gets that $\left|z_{i}\right| \geq \varepsilon>0(i=1,2)$. Then, it readily follows from (11) and (13b) that the matrix $F_{1}^{T}(u, z) F_{1}(u, z)$ remains bounded. Furthermore, this matrix is symmetric nonnegative definite and its determinant $4 E^{2} / \pi^{2} C^{2} L^{2} z_{1}^{2} z_{2}^{2}$ is strictly positive because $\|z\|$ is bounded (by Assumption A3 and (11) and (13b)). The same property can similarly be proved for $F_{k}^{T}(u, z) F_{k}(u, z)$ with $k=2$ and 3 . The Lipschitz feature of the nonlinear functions $G, F_{1}, F_{2}$ and $F_{3}$ is a direct consequence of the smoothness of these functions and the fact that their arguments belong to a compact set, due to Assumption A3

\subsection{Further notations and preliminary computations}

Introduce the time-invariant block diagonal matrix:

$\Delta_{\lambda}=\operatorname{diag}\left[\begin{array}{llll}I_{2 \times 2} & \frac{I_{2 \times 2}}{\lambda} & \frac{I_{2 \times 2}}{\lambda^{2}} & \frac{I_{2 \times 2}}{\lambda^{3}}\end{array}\right]$

where $\lambda>0$ is any positive design parameter. Introduce also the state-dependent block diagonal matrix defined by

$\Lambda(u, z)=\operatorname{diag}\left[I_{2 \times 2} \quad F_{1} \quad F_{1} F_{2} \quad F_{1} F_{2} F_{3}\right]$

On the other hand, it can be checked that

$F^{1} F^{2}=\left(\begin{array}{cc}\frac{4 E}{\pi L^{2} z_{1}} & \frac{2 E u}{\pi L z_{1}} \\ -\frac{2 E}{\pi L C z_{2}} & 0\end{array}\right)$

$F^{1} F^{2} F^{3}=\left(\begin{array}{ll}-\frac{6 E u}{\pi L^{2} z_{1}} & \frac{2 E u}{\pi L C z_{2}}\end{array}\right)^{T}$ 
Then, the left pseudo-inverse of $\Lambda(u, z)$, which exists due to Proposition 1, has the form:

$\Lambda(u, z)^{+}=\operatorname{diag}\left[\begin{array}{llll}I_{2 \times 2} & \left(F_{1}\right)^{+} & \left(F_{1} F_{2}\right)^{+} & \left(F_{1} F_{2} F_{3}\right)^{+}\end{array}\right]$

From (18a), (24) and (25), one has

$F_{1}^{+}=\left(\begin{array}{cc}0 & C z_{2} \\ \frac{-L \pi z_{1}}{2 E} & \frac{-C \pi z_{2}}{2 E}\end{array}\right)$

$\left(F_{1} F_{2}\right)^{+}=\left(\begin{array}{cc}0 & \frac{-L C \pi z_{2}}{2 E} \\ \frac{L \pi z_{1}}{2 E u} & \frac{C \pi z_{2}}{E u}\end{array}\right)$

$\left(F_{1} F_{2} F_{3}\right)^{+}=\left[\begin{array}{ll}\frac{-3}{2 E L^{2} \pi \alpha u z_{1}} & \frac{1}{2 E L C \pi \alpha u z_{2}}\end{array}\right]$

with

$\alpha=\frac{9}{L^{4} \pi^{2} z_{1}^{2}}+\frac{1}{\pi^{2} L^{2} C^{2} z_{2}^{2}}$

Consider the following algebraic Lyapunov equation:

$S_{1}+A^{T} S_{1}+S_{1} A-\bar{C}^{T} \bar{C}=0$

with $A$ and $\bar{C}$ defined as follows:

$A=\left[\begin{array}{cccc}0 & I_{2 \times 2} & 0 & 0 \\ 0 & 0 & I_{2 \times 2} & 0 \\ 0 & 0 & 0 & I_{2 \times 2} \\ 0 & 0 & 0 & 0\end{array}\right], \bar{C}=\left[I_{2 \times 2} 0_{2 \times 2} 0_{2 \times 2} 0_{2 \times 2}\right], \quad$ (due to $(15 b)$ )

It is shown in many places (e.g. Besançon, 2007, Chapter 2) that Eq. (31) has a unique symmetric positive definite matrix solution $S_{1}$ and

$S_{1}^{-1} \bar{C}^{T}=\left[\begin{array}{l}C_{1}^{4} I_{2} \\ C_{2}^{4} I_{2} \\ C_{3}^{4} I_{2} \\ C_{4}^{4} I_{2}\end{array}\right] \quad\left(\right.$ with $\left.C_{m}^{n}=\frac{n !}{m ! \times(n-m) !}\right)$

where $n !=n \times(n-1) \times \cdots \times 2 \times 1$.

\subsection{Observer equations}

Using the above notations, the high-gain state observer for the system (14a)-(14g) is defined as follows:

$\dot{\hat{z}}=F(\hat{z}, u) \hat{z}+G(u, \hat{z})-\lambda \Lambda^{+}(\hat{z}, u) \Delta_{\lambda}^{-1} S_{1}^{-1} \bar{C}^{T}(\bar{C} \hat{z}-y)$

with

$S_{1}^{-1}=\operatorname{col}\left[C_{4}^{1} I_{2} \quad C_{4}^{2} I_{2} \quad C_{4}^{3} I_{2} \quad C_{4}^{4} I_{2}\right]$

$\Delta_{\lambda}^{-1}=\operatorname{diag}\left[I_{2 \times 2} \quad \lambda I_{2 \times 2} \quad \lambda^{2} I_{2 \times 2} \quad \lambda^{3} I_{2 \times 2}\right]$

where $\hat{z}=\left[\hat{z}_{1} \hat{z}_{2} \hat{z}_{3} \hat{z}_{4} \hat{z}_{5} \hat{z}_{6} \hat{z}_{7}\right] \in I R^{7}$ and $S_{1}, \Lambda^{+}(\hat{z}, u), \Delta_{\lambda}, \bar{C}$ are all defined in Section 3.2. The observer (34) is explicitly expressed in terms of the variables $\hat{z}_{i}(i=1, \ldots, 7)$

$\dot{\hat{z}}_{1}=-\frac{\hat{z}_{3}^{2}}{L \hat{z}_{1}}-\frac{2 x_{5}}{n \pi L}-\frac{2 E \hat{z}_{4}}{\pi L \hat{z}_{1}}-4 \lambda\left(\hat{z}_{1}-y_{1}\right)$

$\dot{\hat{z}}_{2}=\frac{\hat{z}_{3}}{C \hat{z}_{2}}-4 \lambda\left(\hat{z}_{2}-y_{2}\right)$

$\dot{\hat{z}}_{3}=-\frac{\hat{z}_{2}^{2}}{L}+\frac{\hat{z}_{1}^{2}}{C}-\frac{2 \hat{z}_{3} x_{5}}{n \pi L \hat{z}_{1}}-\frac{2 E \hat{z}_{5}}{\pi L}-6 \theta^{2} C \hat{z}_{2}\left(\hat{z}_{2}-y_{2}\right)$

$\dot{\hat{z}}_{4}=-\hat{z}_{6} u-\frac{\hat{z}_{5}}{L}-\frac{2 x_{5} \hat{z}_{4}}{n \pi L \hat{z}_{1}}-\frac{2 E}{\pi L}-6 \lambda^{2}\left(\frac{L \pi \hat{z}_{1}}{2 E}\left(\hat{z}_{1}-y_{1}\right)-\frac{C \pi \hat{z}_{2}}{2 E}\left(\hat{z}_{2}-y_{2}\right)\right)$ $\dot{\hat{z}}_{5}=-\hat{z}_{7} u+\frac{\hat{z}_{4}}{C}+4 \lambda^{3} \frac{L C \pi \hat{z}_{2}}{2 E}\left(\hat{z}_{2}-y_{2}\right)$

$\dot{\hat{z}}_{6}=\hat{z}_{4} u-\frac{\hat{z}_{7}}{L}-\frac{2 x_{5} \hat{z}_{6}}{n \pi L \hat{z}_{1}}-\frac{2 E}{\pi L}-6 \lambda^{3}\left(\frac{L \pi \hat{z}_{1}}{2 E u}\left(\hat{z}_{1}-y_{1}\right)-\frac{C \pi \hat{z}_{2}}{E u}\left(\hat{z}_{2}-y_{2}\right)\right)$

$\dot{\hat{z}}_{7}=\hat{z}_{5} u+\frac{\hat{z}_{6}}{C}-\lambda^{4}\left(\frac{\left(\hat{z}_{2}-y_{2}\right)}{2 E \pi L C \hat{z}_{2} \hat{\alpha} u}-\frac{3\left(\hat{z}_{1}-y_{1}\right)}{2 E L^{2} \pi C \hat{z}_{1} \hat{\alpha} u}\right)$

with

$\hat{\alpha}=\frac{9}{L^{4} \pi^{2} \hat{z}_{1}^{2}}+\frac{1}{\left(\pi L C \hat{z}_{2}\right)^{2}}$

\subsection{Observer convergence analysis}

Consider the Lyapunov function

$V_{o b}(\tilde{z})=\tilde{z}^{T} \Delta_{\lambda}^{T} S_{1} \Delta_{\lambda} \tilde{z} \quad$ with $\tilde{z}=z-\hat{z}$

The main result is summarized in the following theorem:

Theorem 1. Consider the system (16) and the observer (34). If the observer gain $\lambda$ is sufficiently large then, whatever the initial condition $\hat{z}(0)$, the time-derivative of $V_{o b}(\tilde{z})$ along the trajectory of $\tilde{z}$ satisfies the inequality

$\dot{V}_{o b} \leq-(\lambda-l) V_{o b}$

for some real constant $l>0$, depending on the Lipschitz coefficients of the different functions i.e. $G, F_{1}, F_{2}$ and $F_{3}$.

The proof relies on the technical properties emphasized in Proposition 1. It can be found in many places, see e.g. Besançon (2007, Chapter 2).

The above theorem shows that the time-derivative of $V_{o b}(\tilde{z})$ can be made negative definite by choosing the observer parameter $\lambda$ sufficiently large so that the condition $\lambda-l>0$ is fulfilled. Then, the state estimation error $\tilde{z}$ vanishes asymptotically, whatever its initial value $\tilde{z}(0)$. This proves the global convergence of the observer (34). A subsidiary consequence of such convergence is that the online state estimates $\hat{z}_{i}(i=1, \ldots, 7)$ remain bounded just as the real variables $\left(z_{1}, \ldots, z_{7}\right)$ do (see Assumption A3).

\section{Adaptive output feedback controller design and analysis}

The load resistance $R$ in the model (1a)-(1c) is unknown but only assumes infrequent jumps. To cope with such a parameter uncertainty the controller must be given a learning capacity. More precisely, the adaptive controller (yet to be designed) will involve an online estimation of the unknown parameter $\theta=1 / R$. The unknown parameter estimate and the corresponding estimation error are denoted $\hat{\theta}$ and $\tilde{\theta}=\theta-\hat{\theta}$, respectively. Three control objectives are sought: (i) asymptotic stability of the closed loop system; (ii) tight regulation of the output voltage $x_{5}=V_{0}$; (iii) as a fast transient response as possible. To this end, an adaptive controller will now be designed in the $z$-coordinates using the backstepping approach. Following closely (Krstic et al., 1995), the control law is designed in three major steps.

Design Step 1. Introduce the tracking error:

$e_{1}=x_{5}-x_{5 r e f}$

where $x_{5 \text { ref }}$ denotes the desired constant-like output reference. Achieving the tracking objective amounts to enforcing the error $e_{1}$ to vanish. To this end, the $e_{1}$-dynamics need to be clearly defined. Deriving (40) one obtains:

$\dot{e}_{1}=\frac{4}{n \pi C_{o}} z_{1}-\frac{x_{5}}{C_{o}} \theta$ 
where the quantity $\frac{4}{n \pi C_{0}} z_{1}$ stands as a virtual control input. Consider the following Lyapunov function:

$V_{c 1}\left(e_{1}, \tilde{\theta}\right)=\frac{1}{2} e_{1}^{2}+\frac{1}{2 \gamma} \tilde{\theta}^{2}$

where $\gamma>0$ is a design parameter, called adaptation gain. Deriving $V_{c 1}$ along the $\left(e_{1}, \tilde{\theta}\right)$-trajectory gives

$\dot{V}_{c 1}=e_{1}\left(\frac{4}{n \pi C_{o}} z_{1}+w_{1} \hat{\theta}\right)-\frac{\tilde{\theta}}{\gamma}\left(\dot{\hat{\theta}}-\gamma w_{1} e_{1}\right)$

where $w_{1}$ denotes the first regressor function defined by

$w_{1}=-\frac{x_{5}}{C_{0}}$

One could eliminate $\tilde{\theta}$ from $\dot{V}_{1}$ using the following parameter update law:

$\dot{\hat{\theta}}=\gamma \tau_{1}$

with

$\tau_{1}=w_{1} e_{1}$

Furthermore, $e_{1}$ could be regulated to zero by letting $\frac{4}{n \pi C_{0}} z_{1}=\alpha_{1}$, where the stabilizing function $\alpha_{1}$ is defined by

$\alpha_{1}=-c_{1} e_{1}-w_{1} \hat{\theta}$

where $c_{1}>0$ is a design parameter. Since $\frac{4 z_{1}}{n \pi c_{0}}$ is not the actual control input, one can only seek the convergence of the error $\frac{4 z_{1}}{n \pi c_{0}}-\alpha_{1}$ to zero. Also, $\hat{\theta}=\gamma \tau_{1}$ is not kept as the parameter update law. Nevertheless, $\tau_{1}$ is retained as the first tuning function and the presence of $\tilde{\theta}$ in $\dot{V}_{1}$ is tolerated. Therefore, the following second error variable is defined:

$e_{2}=\frac{4}{n \pi C_{o}} z_{1}-\alpha_{1}$

Then, using (47) and (48), Eq. (41) becomes

$\dot{e}_{1}=-c_{1} e_{1}+e_{2}+w_{1} \tilde{\theta}$

Also, (43) can be rewritten as follows:

$\dot{V}_{c 1}=-c_{1} e_{1}^{2}+e_{1} e_{2}+\tilde{\theta}\left(\tau_{1}-\frac{\dot{\hat{\theta}}}{\gamma}\right)$

Design Step 2. Now, the objective is to make the error variables $\left(e_{1}, e_{2}\right)$ vanish asymptotically. To this end, the dynamics of $e_{2}$ are first determined. Deriving (48) one obtains, using (14a)-(14g), (45), (47) and (49):

$$
\begin{aligned}
\dot{e}_{2}= & -\frac{8 E z_{4}}{n \pi^{2} L C_{0} z_{1}}-\frac{4 z_{3}}{n \pi L C_{0} z_{1}}-\frac{8 x_{5}}{n^{2} \pi^{2} L C_{o}}-\frac{\hat{\theta}}{C_{o}}\left(\frac{4}{n \pi C_{o}} z_{1}-\frac{x_{5}}{C_{o}} \hat{\theta}\right) \\
& +c_{1}\left(-c_{1} e_{1}+e_{2}\right)+w_{1} \dot{\hat{\theta}}+\tilde{\theta}\left(c_{1} w_{1}+\frac{x_{5}}{C_{o}^{2}} \hat{\theta}\right)
\end{aligned}
$$

As the states $z_{i}(i=3,4)$ are not available they are replaced in (51) by their estimates, provided by (37a)-(37h). Doing so, one gets:

$$
\begin{aligned}
\dot{e}_{2}= & -\frac{8 E \hat{z}_{4}}{n \pi^{2} L C_{o} z_{1}}-\frac{4 \hat{z}_{3}}{n \pi L C_{o} z_{1}}-\frac{8 x_{5}}{n^{2} \pi^{2} L C_{o}} \\
& -\frac{\hat{\theta}}{C_{o}}\left(\frac{4}{n \pi C_{o}} z_{1}-\frac{x_{5}}{C_{o}} \hat{\theta}\right)+c_{1}\left(-c_{1} e_{1}+e_{2}\right) \\
& +w_{1} \dot{\hat{\theta}}+\tilde{\theta}\left(c_{1} w_{1}+\frac{x_{5}}{C_{o}^{2}} \hat{\theta}\right)-\frac{8 E}{n \pi^{2} L C_{0}} \frac{\tilde{z}_{4}}{z_{1}}-\frac{4}{n \pi L C_{0}} \frac{\tilde{z}_{3}}{z_{1}}
\end{aligned}
$$

where $\tilde{z}_{3}$ and $\tilde{z}_{4}$ are the estimation errors of $z_{3}$ and $z_{4}$. The quantity $-\left(8 E \hat{z}_{4} / n \pi^{2} L C_{o} z_{1}\right)$ stands in (52) as a virtual control. Let $\alpha_{2}$ denote the corresponding stabilizing function, yet to be found and introduce the new error

$e_{3}=-\frac{8 E \hat{z}_{4}}{n \pi^{2} L C_{0} z_{1}}-\alpha_{2}$
Then (52) is rewritten as follows:

$\dot{e}_{2}=e_{3}+\alpha_{2}+\psi_{2}+w_{2} \tilde{\theta}+w_{1} \dot{\hat{\theta}}+\chi_{1}\left(z_{1}, \tilde{z}_{3}, \tilde{z}_{4}\right)$

where the second regression function is defined by

$w_{2}=c_{1} w_{1}+\frac{x_{5}}{C_{0}^{2}} \hat{\theta}$

and

$\psi_{2}=-\frac{4}{n \pi L C_{0} z_{1}} \hat{z}_{3}-\frac{8 x_{5}}{n^{2} \pi^{2} L C_{0}}+c_{1}\left(-c_{1} e_{1}+e_{2}\right)-\frac{\hat{\theta}}{C_{0}}\left(\frac{4}{n \pi C_{0}} z_{1}-\frac{x_{5}}{C_{o}} \hat{\theta}\right)$

$\chi_{1}\left(z_{1}, \tilde{z}_{3}, \tilde{z}_{4}\right)=-\frac{8 E}{n \pi^{2} L C_{0}} \frac{\tilde{z}_{4}}{z_{1}}-\frac{4}{n \pi L C_{0}} \frac{\tilde{z}_{3}}{z_{1}}$

Notice that the disturbing term $\chi_{1}\left(z_{1}, \tilde{z}_{3}, \tilde{z}_{4}\right)$ vanishes exponentially fast whenever $\tilde{z}_{3}, \tilde{z}_{4}$ do so. Consider the augmented Lyapunov function:

$V_{c 2}\left(e_{1}, e_{2}, \tilde{\theta}\right)=V_{c 1}\left(e_{1}, \tilde{\theta}\right)+\frac{1}{2} e_{2}^{2}$

Its derivative along the solution of (40) and (48) is

$\dot{V}_{c 2}=-c_{1} e_{1}^{2}+e_{2}\left(e_{1}+e_{3}+\alpha_{2}+\psi_{2}+w_{1} \dot{\hat{\theta}}\right)+\tilde{\theta}\left(\tau_{1}+e_{2} w_{2}-\frac{\dot{\hat{\theta}}}{\gamma}\right)+e_{2} \chi_{1}$

This shows that $\tilde{\theta}$ can be canceled in $\dot{V}_{2}$ using the update law $\dot{\hat{\theta}}=\gamma \tau_{2}$

with

$\tau_{2}=\tau_{1}+\omega_{2} e_{2}=\left[\begin{array}{ll}w_{1} & w_{2}\end{array}\right]\left[\begin{array}{l}e_{1} \\ e_{2}\end{array}\right]$

If $\left(-\frac{8 E \hat{z}_{4}}{n \pi^{2} L C_{0} z_{1}}\right)$ were the actual control in (52) and the term $\chi_{1}$ were null, then (59) would reduce to $\dot{V}_{c 2}=-c_{1} e_{1}^{2}-c_{2} e_{2}^{2}$ by using the above parameter update law and the stabilizing function

$\alpha_{2}=-e_{1}-\psi_{2}-c_{2} e_{2}-w_{1} \gamma \tau_{2}$

As $\left(-\frac{8 E \hat{z}_{4}}{n \pi^{2} L C_{0} z_{1}}\right)$ is just a virtual control, the above parameter update law is not sufficient. Nevertheless, $\tau_{2}$ is retained as a second tuning function. Then, (59) gives

$\dot{V}_{c 2}=-c_{1} e_{1}^{2}-c_{2} e_{2}^{2}-e_{2} w_{1}\left(\gamma \tau_{2}-\dot{\hat{\theta}}\right)+\tilde{\theta}\left(\tau_{2}-\frac{\dot{\hat{\theta}}}{\gamma}\right)+e_{2} e_{3}+e_{2} \chi_{1}\left(z_{1}, \tilde{z}_{3}, \tilde{z}_{4}\right)$

On the other hand, in view of (49), (54), (56) and (61), the $\left(e_{1}, e_{2}\right)$-system undergoes the following equation:

$$
\begin{aligned}
{\left[\dot{e}_{1} \dot{e}_{2}\right]=} & {\left[\begin{array}{cc}
-c_{1} & 1 \\
-1 & -c_{2}
\end{array}\right]\left[\begin{array}{l}
e_{1} \\
e_{2}
\end{array}\right]+\left[\begin{array}{c}
0 \\
e_{3}-w_{1}\left(\gamma \tau_{2}-\dot{\hat{\theta}}\right)
\end{array}\right] } \\
& +\left[\begin{array}{l}
w_{1} \\
w_{2}
\end{array}\right] \tilde{\theta}+\left[\begin{array}{c}
0 \\
\chi_{1}\left(z_{1}, \tilde{z}_{3}, \tilde{z}_{4}\right)
\end{array}\right]
\end{aligned}
$$

Design Step 3. Here the objective is to make all control errors $\left(e_{1}, e_{2}, e_{3}\right)$ vanish asymptotically. Deriving (53) gives

$\dot{e}_{3}=-\frac{8 E}{n \pi^{2} L C_{o}} \overbrace{\left(\frac{\hat{z}_{4}}{z_{1}}\right)}^{i}-\dot{\alpha}_{2}$

On the other hand, one obtains from (14a) and (37d)

$\overbrace{\left(\frac{\hat{z}_{4}}{z_{1}}\right)}=-\frac{\hat{z}_{6}}{z_{1}} u+\delta_{1}\left(z_{1}, z_{2}, \hat{z}\right)+\tilde{z}_{3} \frac{\hat{z}_{4}}{L z_{1}^{3}}+\tilde{z}_{4} \frac{2 E \hat{z}_{4}}{\pi L z_{1}^{3}}$ 
with

$$
\begin{aligned}
\delta_{1}\left(z_{1}, z_{2}, \hat{z}\right)= & -\left(\frac{\hat{z}_{5}}{L z_{1}}+2 \frac{x_{5} \hat{z}_{4}}{n \pi L z_{1}^{2}}+\frac{2 E}{\pi L z_{1}}-\frac{\hat{z}_{4}}{z_{1}^{2}}\left(-\frac{\hat{z}_{3}}{L z_{1}}-\frac{2 x_{5}}{n \pi L}-\frac{2 E}{\pi L z_{1}} \hat{z}_{4}\right)\right) \\
& +\frac{6 \lambda^{2}}{z_{1}}\left(\frac{L \pi \hat{z}_{1}}{2 E}\left(\hat{z}_{1}-z_{1}\right)-\frac{C \pi \hat{z}_{2}}{2 E}\left(\hat{z}_{2}-z_{2}\right)\right)
\end{aligned}
$$

Furthermore, it is readily seen from (61) that

$\dot{\alpha}_{2}=-\gamma\left[\dot{w}_{1} \tau_{2}+w_{1}\left(\dot{w}_{1} e_{1}+w_{1} \dot{e}_{1}+\dot{w}_{2} e_{2}+w_{2} \dot{e}_{2}\right)\right]-\dot{e}_{1}-\dot{\psi}_{2}-c_{2} \dot{e}_{2}$

Using (44), (55), (56), (57) and (63), the derivatives on the right side of (67) can be given the following more suitable form:

$\dot{e}_{1}=e_{10}+w_{1} \tilde{\theta}$

$\dot{e}_{2}=e_{20}+w_{1} \dot{\hat{\theta}}+w_{2} \tilde{\theta}+\chi_{1}(\cdot)$

$\dot{w}_{1}=-\frac{\chi_{50}}{C_{o}}-\frac{w_{1}}{C_{o}} \tilde{\theta}$

$\dot{\psi}_{2}=a_{0}\left(z_{10}+\tilde{z}_{10}\right)+a_{1} \dot{x}_{5}-a_{2} \frac{\dot{\hat{\theta}}}{C_{0}}+c_{1}\left(-c_{1} \dot{e}_{1}+\dot{e}_{2}\right)-\frac{4 \dot{\hat{z}}_{3}}{n \pi L C_{0} z_{1}}$

To alleviate the text, the exact expressions of the newly introduced quantities (i.e. $e_{10}, e_{20}, x_{50}, z_{10}, \tilde{z}_{10}, a_{0}, a_{1}$ and $a_{2}$ ) are placed in Appendix A. Substituting (65) and (67) in (64), one obtains

$\dot{e}_{3}=\frac{8 E \hat{z}_{6}}{n \pi^{2} L C_{o} z_{1}} u+\delta_{2}(z, \hat{z})+w_{3} \tilde{\theta}+g_{3} \dot{\hat{\theta}}+\chi_{2}\left(e_{1}, e_{2}, e_{3}, z, \tilde{z}\right)$

where $w_{3}$ denotes the last regression function defined by:

$w_{3}=\left(1-c_{1}^{2}+\gamma w_{1}^{2}\right)+\left(c_{1}+c_{2}+\gamma w_{1} w_{2}\right) w_{2}$

$$
-\gamma\left(\tau_{2}+w_{1} e_{1}+w_{1} e_{2}\left(c_{1}-\frac{\hat{\theta}}{C_{0}}\right)-C_{o} a_{1}\right) w_{1}
$$

$$
\begin{aligned}
\delta_{2}=- & \frac{8 E}{n \pi^{2} L C_{0}} \delta_{1}+\left(1-c_{1}^{2}+\gamma w_{1}^{2}\right) e_{10}+a_{0} z_{10} \\
& -\frac{4}{\pi L C_{0} z_{1}} \dot{\hat{z}}_{3}+\left(c_{1}+c_{2}+\gamma w_{1} w_{2}\right) e_{20} \\
& -\frac{\gamma}{C_{0}} x_{50}\left(\tau_{2}+w_{1} e_{1}+w_{1} e_{2}\left(c_{1}-\frac{\hat{\theta}}{C_{o}}\right)-a_{1} C_{0}\right)
\end{aligned}
$$

$g_{3}=w_{1}\left(c_{1}+c_{2}+\gamma w_{1} w_{2}\right)-\left(\frac{a_{2}}{C_{o}}+\gamma \frac{w_{1}^{2} e_{2}}{C_{o}}\right)$

$\chi_{2}=-\frac{8 E}{n \pi^{2} L C_{o}}\left(\tilde{z}_{3} \frac{\hat{z}_{4}}{L z_{1}^{3}}+\tilde{z}_{4} \frac{2 E \hat{z}_{4}^{2}}{\pi L z_{1}^{3}}\right)+\left(c_{1}+c_{2}+\gamma w_{1} w_{2}\right) \chi_{1}+a_{0} \tilde{z}_{10}$

Note that the actual control input $u$ has emerged for the first time in (72). Notice also that the term in $\chi_{2}$ vanishes exponentially fast whenever the $\tilde{z}_{3}, \tilde{z}_{4}$ do so. Now, the goal is to find a control law for $u$ and an update law for $\hat{\theta}$ so that the $\left(e_{1}, e_{2}, e_{3}, \tilde{\theta}\right)$ system is asymptotically stable. To this end, consider the augmented Lyapunov function candidate

$V_{c 3}\left(e_{1}, e_{2}, e_{3}, \tilde{\theta}\right)=V_{c 2}\left(e_{1}, e_{2}, \tilde{\theta}\right)+\frac{e_{3}^{2}}{2}=\sum_{i=1}^{3} \frac{e_{i}^{2}}{2}+\frac{\tilde{\theta}^{2}}{2 \gamma}$

Using (62) and (72), the derivative of $V_{c 3}$ turns out to be

$$
\begin{aligned}
\dot{V}_{c 3}= & -c_{1} e_{1}^{2}-c_{2} e_{2}^{2}-e_{2} w_{1}\left(\gamma \tau_{2}-\dot{\hat{\theta}}\right)+e_{3}\left[\frac{8 E \hat{z}_{6}}{n \pi^{2} L C z_{1}} u+\delta_{2}+g_{3} \dot{\hat{\theta}}+e_{2}\right] \\
& +\tilde{\theta}\left(\tau_{2}-\frac{\dot{\hat{\theta}}}{\gamma}+e_{3} w_{3}\right)+e_{3} \chi_{2}\left(e_{1}, e_{2}, e_{3}, \tilde{z}, z\right)
\end{aligned}
$$

The term in $\tilde{\theta}$ could be canceled on the right side of (78) using the following update law:

$\dot{\hat{\theta}}=\gamma \tau_{3}$

with

$\tau_{3}=\tau_{2}+e_{3} w_{3}=\left[\begin{array}{lll}w_{1} & w_{2} & w_{3}\end{array}\right]\left[\begin{array}{l}e_{1} \\ e_{2} \\ e_{3}\end{array}\right]=w e$

$e=\left[\begin{array}{lll}e_{1} & e_{2} & e_{3}\end{array}\right]^{T}$ and $w=\left[\begin{array}{lll}w_{1} & w_{2} & w_{3}\end{array}\right]$

However, this update law (which is a gradient type) is not suitable because of its integral nature. The disturbing term $\chi_{2}(z, \tilde{z}, \hat{z})$ in (78) may cause the divergence the estimate $\hat{\theta}$. This issue is commonly coped with resorting to estimate projection on a convex compact set including the true parameter (e.g. Ioannou and Fidan, 2006; El Fadil and Giri, 2009). Let such convex be any interval $C=\left[-M_{0}, M_{0}\right]$ such that $M_{0} \geq|\theta|$. Practical choice of $M_{0}$ is not an issue as this may be arbitrarily large. The gradient algorithm with projection is then defined as follows:

$\dot{\hat{\theta}}=P\left(\gamma \tau_{3}\right)$

where $\hat{\theta}(0)$ is chosen so that $\hat{\theta}^{2}(0) \leq M_{0}^{2}, P(\cdot)$ is the projection operator defined by

$P\left(\gamma \tau_{3}\right) \stackrel{\text { def }}{=} \begin{cases}\gamma \tau_{3} & \text { if } \hat{\theta}^{2}<M_{0}^{2} \text { or if }\left(\hat{\theta}^{2}=M_{0}^{2} \text { and } \gamma \tau_{3} \hat{\theta} \leq 0\right) \\ 0 & \text { otherwise }\end{cases}$

It is readily seen that this adaptive law maintains the estimate $\hat{\theta}$ within the convex bounded set $C$. More interestingly, the projection operator $P(\cdot)$ is shown in many places to possess the following key property (see e.g. Ioannou \& Fidan, 2006):

$-\tilde{\theta} P\left(\gamma \tau_{3}\right) \leq-\tilde{\theta} \gamma \tau_{3}$

Owing to the control action $u$, the expression of $\dot{V}_{c 3}$ suggests the following control law:

$u=\frac{n \pi^{2} L C_{0} z_{1}}{8 E \hat{z}_{6}}\left(-\delta_{2}-g_{3} \dot{\hat{\theta}}-e_{2}-c_{3} e_{3}+v\right)$

where $c_{3}>0$ is a new parameter and $v$ is an additional control action resorted to cope with the parameter adaptive law saturation. The following choice will prove to be useful:

$v=\left\{\begin{array}{lll}-\gamma w_{1} w_{3} e_{2} & \text { if } & \dot{\hat{\theta}}=\gamma \tau_{3} \\ 0 & \text { if } & \dot{\hat{\theta}}=0\end{array}\right.$

The adaptive output feedback controller thus established is composed of the control law (83) and (84), the parameter update law (80) and (81) and the state observer (34) (or (37a)-(37h)). Substituting the right side of (83) to $u(t)$ in (72) and putting the resulting equation together with (68), (69) and (80), one gets the following equations describing the closed-loop control system, in terms of the errors $e$ and $\tilde{\theta}$ :

$\dot{e}=\bar{A} e+w^{T} \tilde{\theta}+\overleftarrow{w}+\chi$

$\dot{\tilde{\theta}}=-\dot{\hat{\theta}}=-P\left(\gamma \tau_{3}\right)$ 
where $\bar{A}$ is a skew symmetric matrix defined by

$\bar{A}=\left[\begin{array}{ccc}-c_{1} & 1 & 0 \\ -1 & -c_{2} & 1+\sigma_{23} \\ 0 & -1-\sigma_{23} & -c_{3}\end{array}\right], \quad\left(\right.$ with $\left.\sigma_{23}=\gamma w_{1} w_{3}\right)$

and

$\overleftarrow{w}=\left[\begin{array}{ll}0 & -w_{1}\left(\gamma \tau_{2}-\dot{\hat{\theta}}\right) v\end{array}\right]^{T}$

$\chi=\left[\begin{array}{lll}0 & \chi_{1}(\cdot) & \chi_{2}(\cdot)\end{array}\right]^{T}$

The performances of the closed-loop system will now be described in the next theorem, using the following usual notations (Khalil, 2003):

- $\|x\|$ denotes the Euclidean norm of any finite dimension vector $x$,

- if $x$ is a function of time then $\|x\|_{2}$ and $\|x\|_{\infty}$ denote, respectively, its $L_{2}$ and $L_{\infty}$ norms:

$\|x\|_{2}=\int_{0}^{\infty}\|x(t)\|^{2} d t, \quad\|x\|_{\infty}=\sup _{0 \leq t<\infty}\|x(t)\|$

The set of all functions $x$ such that $\|x\|_{p}<\infty$ is denoted $L_{p}\left(\begin{array}{ll}0 & \infty\end{array}\right)$ (or simply $\left.L_{p}\right)$, with $p \in\{2, \infty\}$.

Theorem 2. (Main result). Consider the control system consisting of the SRC model (1a)-(1c) in closed-loop with the adaptive controller composed of the control law (83) and (84), the parameter update law (80) and (81) and the high gain observer defined by (37a)-(37h). There exists a real constant $c_{\min }>0$ such that, if $\min \left(c_{1}, c_{2}, c_{3}\right)>c_{\min }$ then:

1) all closed-loop signals remain bounded,

2 ) the output reference tracking error $e_{1}=x_{5}-x_{5 \text { ref }}$ belongs to $L_{2}$ and vanishes asymptotically.

Remarks 2. (a) The first part of the theorem ensures that all auxiliary signals, introduced throughout the regulator design steps, remain bounded. This particularly applies to the control signal $u$ and $\hat{\theta}$. The second part ensures that the output voltage tracking error actually vanishes asymptotically with an $L_{2}$ rate convergence i.e. $e_{1}^{2}(t)$ converges to zero at least as rapidly as $1 / t^{p}$, with $p \geq 2$, when $t \rightarrow \infty$. The proof of the theorem is a bit long and so is placed in Appendix B alleviating thus the presentation.

(b) Robust control could be an alternative to adaptive control in coping with load uncertainty. In this respect, note that a robust controller could be obtained using the (nonadaptive) backstepping control design technique that involves damping functions (Krstic et al., 1995). This control strategy was used (to cope with similar load uncertainties) in previous works e.g. (El Fadil, Giri, El Maguiri, \& Chaoui, 2009; El Magri, Giri, Abouloifa, \& Chaoui, 2010).

\section{Simulation results}

The controlled system is represented by Eqs. (1a)-(1c) where the different components have the numerical values of Table 1. The DC voltage source is fixed to $E=60 \mathrm{~V}$. The adaptive output feedback controller, including the control law (83) and (84), the parameter update law (80) and (81) and the state observer (34), is given the following design parameters that proved to be convenient:

$\lambda=1 \times 10^{3}, \quad c_{1}=45 \times 10^{2}, \quad c_{2}=1 \times 10^{2}$,

$c_{3}=1.9 \times 10^{2}, \quad \gamma^{-1}=1 \times 10^{16}$ and $M_{0}=10$.
Table 1

Numerical values of the SRC characteristics.

\begin{tabular}{llll}
\hline Parameters & Symbols & Values & Additional information \\
\hline Resonant inductor & $L$ & $0.9 \times 10^{-3} \mathrm{H}$ & Ferrite core coil \\
Resonant capacitor & $C$ & $130 \times 10^{-6} \mathrm{~F}$ & Film capacitor \\
Output capacitor & $C_{o}$ & $2.4 \times 10^{-3} \mathrm{~F}$ & Electrolytic capacitor \\
Isolation transform & $T$ & Turn ratio 1 & Ferrite core coil \\
\hline
\end{tabular}

The initial value of the state vectors and parameter estimates are chosen as follows:

$x(0)=\left[\begin{array}{lllll}0.35 & -0.75 & -5 & -8 & 10\end{array}\right]^{T}$

$\hat{z}_{1}(0)=0.3, \quad \hat{z}_{2}(0)=0.3, \quad \hat{z}_{3}(0)=0.2, \quad \hat{z}_{4}(0)=0$,

$\hat{z}_{5}(0)=0, \quad \hat{z}_{6}(0)=0.25, \quad \hat{z}_{7}(0)=0.5, \quad \hat{z}_{8}(0)=0$

Recall that the correspondence between the sate vectors $x$ and $z$ is defined by (13b) which is rewritten for convenience:

$z_{1}=\sqrt{x_{1}^{2}+x_{2}^{2}}, \quad z_{2}=\sqrt{x_{3}^{2}+x_{4}^{2}}, \quad z_{3}=x_{1} x_{3}+x_{2} x_{4}$,

$z_{4}=x_{2}, \quad z_{5}=x_{4}, \quad z_{6}=x_{1}, \quad z_{7}=x_{3}$

\subsection{First harmonic model checking}

Here, the aim is to check the good accuracy of the (first harmonic approximation) model defined by Eqs. (6)-(8) (or equally (10a)-(10e)) in approximating the behavior of the real-life system represented by the (more physical) model (1)-(3). In this respect, recall that the variables $I_{1}, V_{0}$ and $V_{1}$ in the model (6)-(8) represent by definition the (time-varying) amplitudes of the variables $i(t), v_{0}(t)$ and $v(t)$ in the model (1)-(3). In other words, $I_{1}, V_{0}$ and $V_{1}$ are nothing other than the envelops of $i(t), v_{0}(t)$ and $v(t)$, respectively. Both models are simulated, in the Matlab/Simulink environment, using the numerical parameter values, $f_{s}=3 \mathrm{Khz}, R=2.3 \Omega$. The resulting variables are shown by Fig. $3 \mathrm{a}-\mathrm{c}$. It is seen that $I_{1}, V_{0}$ and $V_{1}$ are actually very close to the envelops of $i(t), v_{0}(t)$ and $v(t)$. Therefore, the model (10a)-(10e) can be based upon to control the physical circuit represented by (1)-(3).

\subsection{Controller performances in presence of varying load}

The controller performances are illustrated by Figs. 4-8. Fig. 4 illustrates the closed-loop system responses to a step reference $x_{5 r e f}=48 \mathrm{~V}$ (stepping time $t=0$ ) in presence of converter load changes. Fig. 5 shows that the true load jumps between 2.3 and $4.6 \Omega$ (resulting in nearly $50 \%$ in power change). It is seen from Fig. 4 that the output voltage is well regulated as it shortly recovers its reference value after each transient periods following load changes. Fig. 5 shows that the load estimate $\hat{\theta}^{-1}$ does converge toward its true value $\theta^{-1}=R$. The resulting control frequency is shown by Fig. 6 . Fig. 7 shows the trajectory of all state estimates obtained with $\lambda=1000$. It is seen that the trajectories of the true states $\left(x_{1}, x_{2}, x_{3}, x_{4}\right)$ and those of their estimates are too close to be distinguished. Then, a zoom is made on the state estimation errors in Fig. 8 showing that the errors vanish after $6 \mathrm{~ms}$. Note that the convergence rate depends on the value of the observer gain $\lambda$.

\subsection{Controller performances in presence of system parameters uncertainties}

In this subsection, the true converter parameters are subject to uncertainty, but the controller design is still based on the converter nominal values of Table 1 . In all simulated experiments, 
a
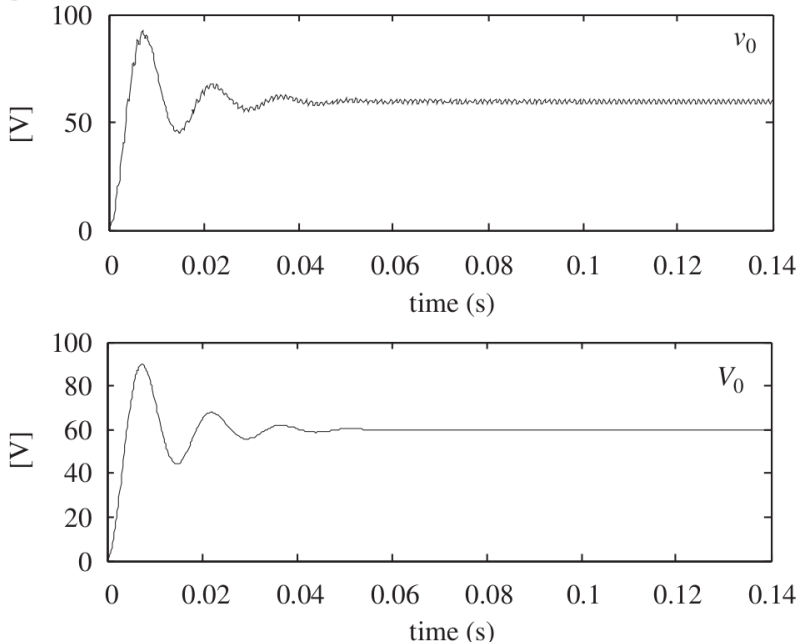

b
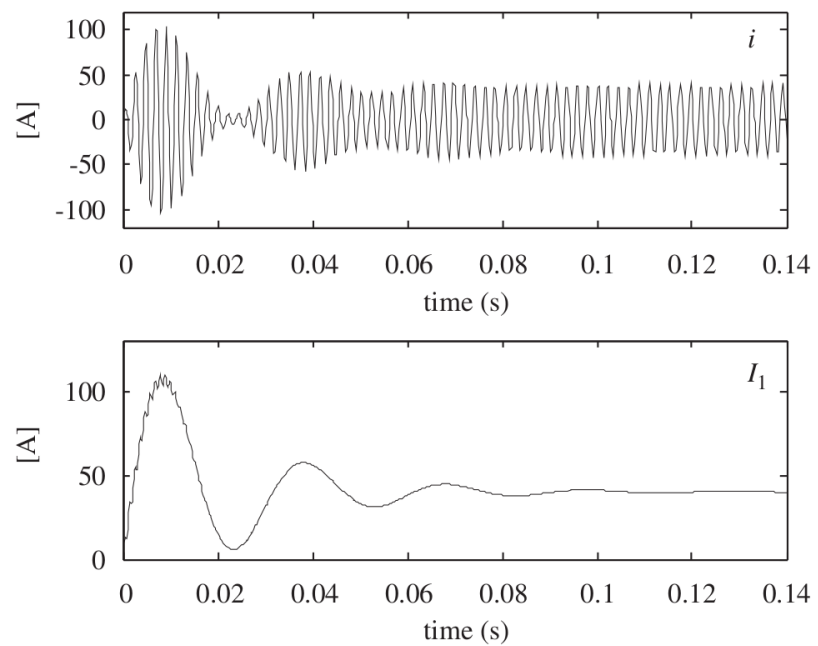

C
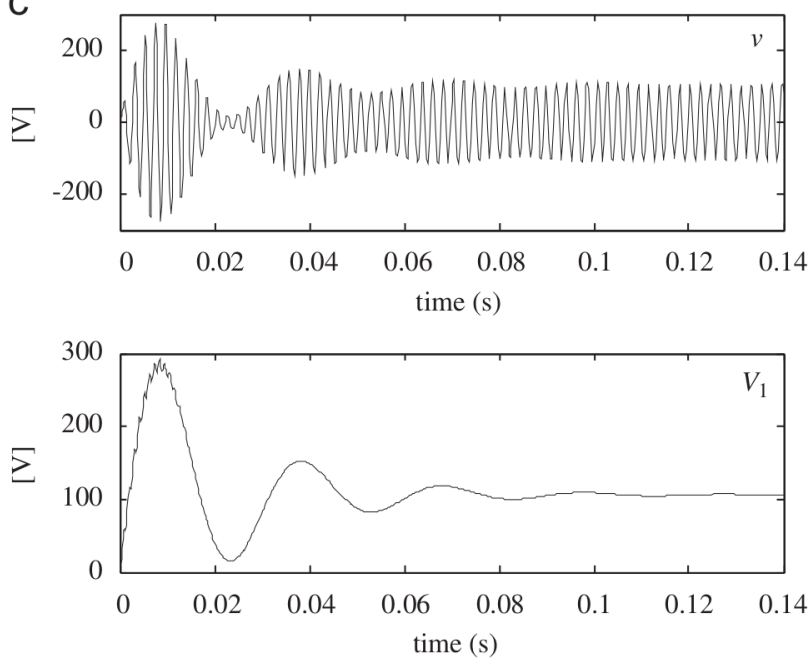

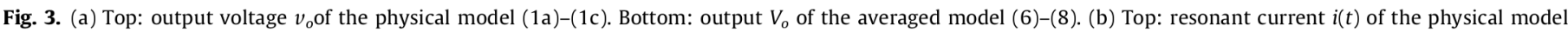

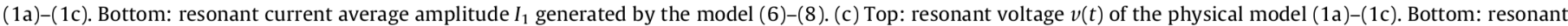
voltage amplitude $V_{1}$ generated by the model (6)-(8).

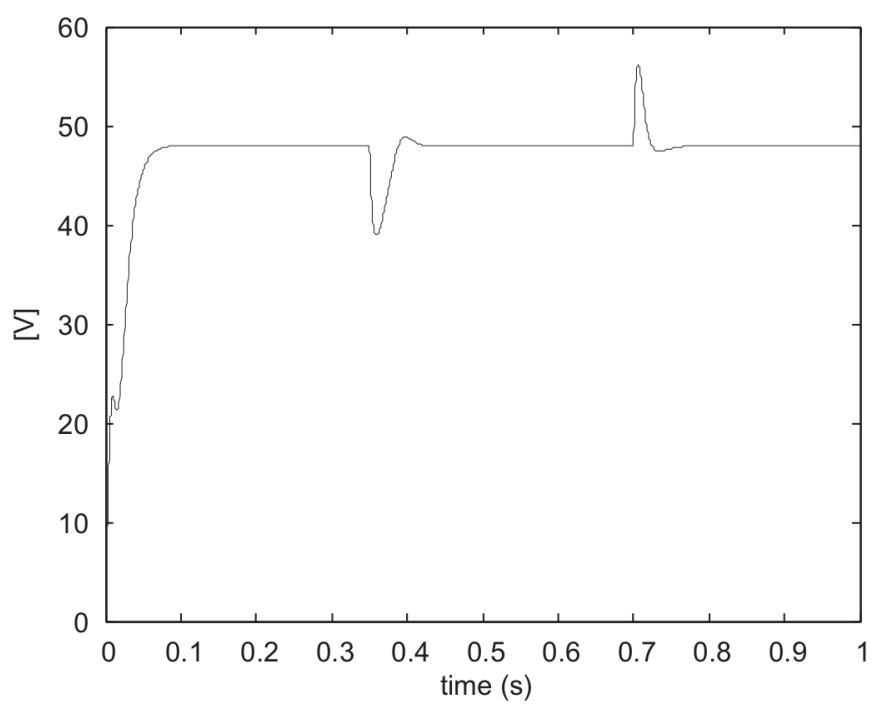

Fig. 4. Output voltage regulation in presence of varying converter load.

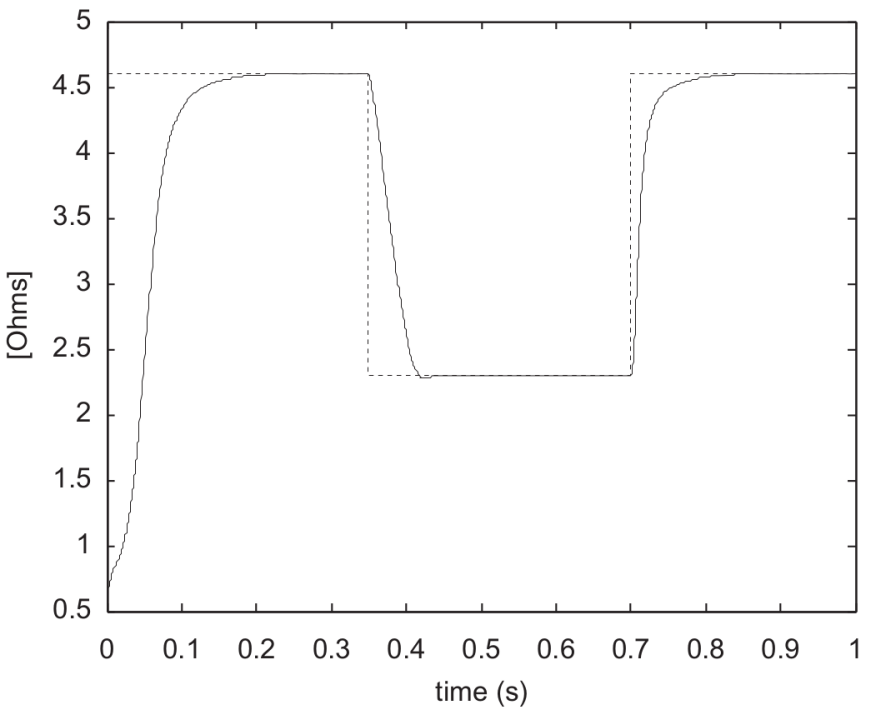

Fig. 5. Varying converter load estimation: true load $R=\theta^{-1}$ (dashed) and its estimate $\hat{R}=\hat{\theta}^{-1}$ (solid). 
the controller design parameters are given the following values that prove to be suitable:

$\lambda=1 \times 10^{3}, \quad c_{1}=30 \times 10^{2}, \quad c_{2}=1 \times 10^{2}, \quad c_{3}=1.9 \times 10^{2}$,

$\gamma^{-1}=1 \times 10^{16}$, and $M_{0}=10$.

\subsection{Controller behavior in presence of disturbed and changing voltage source}

In the first experiment, the source voltage uncertainty $\Delta E(t)$ is let to be time-varying according to the following protocol:

- in the time interval $[0,4 \mathrm{~ms}]: \Delta E(t)$ is a zero mean random noise, with variance $0.3 \mathrm{~V}^{2}$,

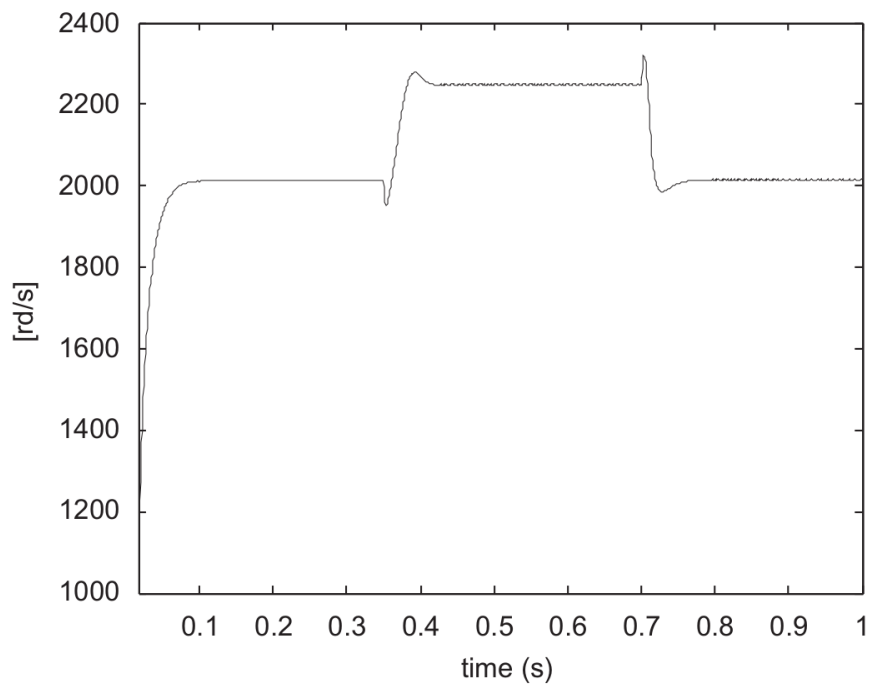

Fig. 6. Control frequency.
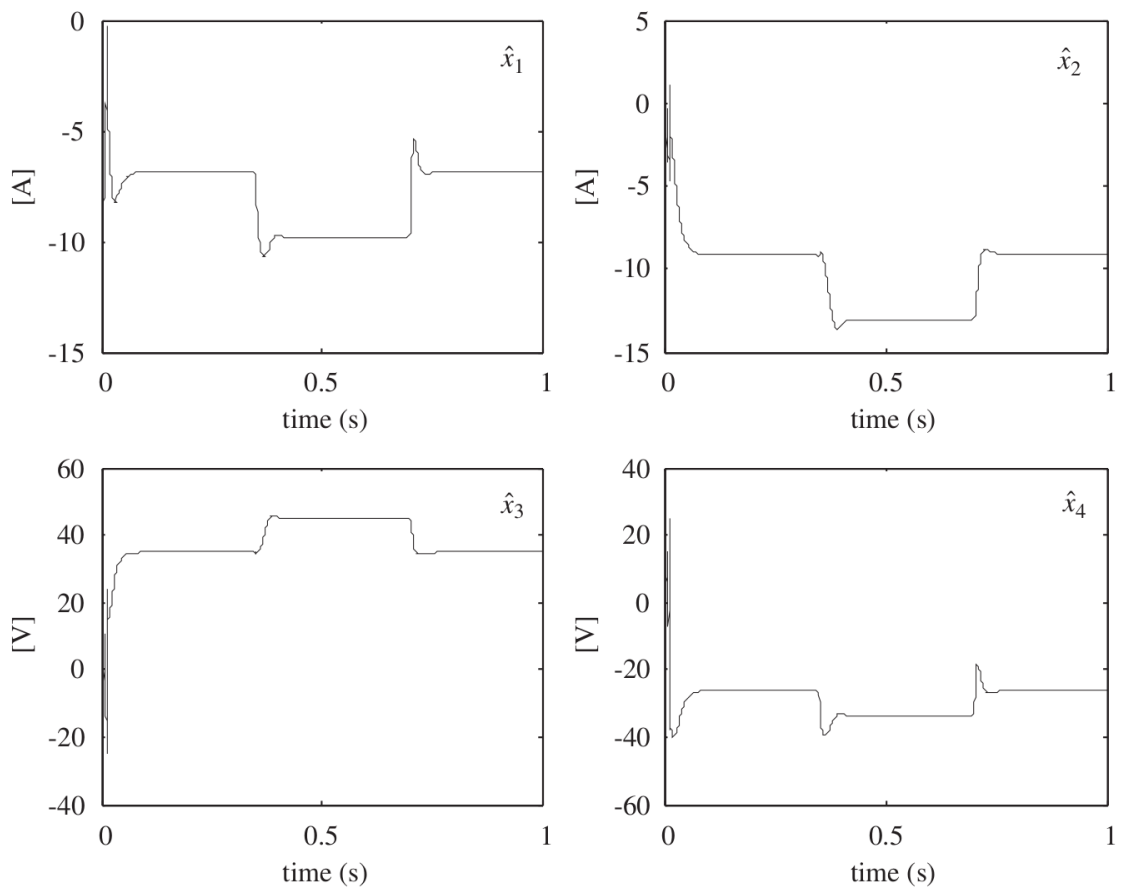

- from time 4 to $7 \mathrm{~ms}: \Delta E(t)=4 \mathrm{~V}$ (step like change),

- from time $7 \mathrm{~ms}$ to the end: $\Delta E(t)=-5 \mathrm{~V}$.

The resulting voltage source variation is shown in Fig. 9 (top). In this experiment, the load resistance and the output voltage reference are both kept constant:

$R=2.3 \Omega, \quad v_{0 \text { ref }}=x_{5 \text { ref }}=48 \mathrm{~V}$.

Fig. 9 shows that the controller compensates well the disturbing effect due to source voltage uncertainty and variation. That is, the proposed controller is quite insensitive to voltage source uncertainties.

\subsection{Controller behavior in presence of uncertainties on $(L, C)$}

In this subsection, the converter capacitance and inductance are subject to uncertainty, namely $\Delta L=0.045 \mathrm{mH}, \Delta C=6.5 \mu \mathrm{F}$. These uncertainties represent $5 \%$ of the parameter nominal values. Presently, the unknown load resistance is fixed to $R=2.3$ $\Omega$ and the output voltage reference trajectory $V_{0 r e f}=x_{5 \text { ref }}$ is let to be a filtered step-like signal taking the values 49,54 and $46 \mathrm{~V}$. Filtering is performed by a third order linear filter; it is resorted to make $V_{0 r e f}=x_{5 \text { ref }}$ time-derivable with known derivatives (because these are needed in the control law). The resulting controller performances are illustrated by Fig. 10 which shows that:

- the load estimate $\hat{R}=\hat{\theta}^{-1}=2.26 \Omega$ matches its true value up to a small error of $1.81 \%$,

- the output voltage $V_{0}=x_{5}$ tracks well its reference, up to a relative error not exceeding $2.5 \%$.

That is, the output tracking error (less that 2.5\%) is much smaller that the parameter uncertainty (5\%). This proves again the controller robustness against parameter uncertainties.

Fig. 7. State variable trajectories with $\lambda=1000$. 

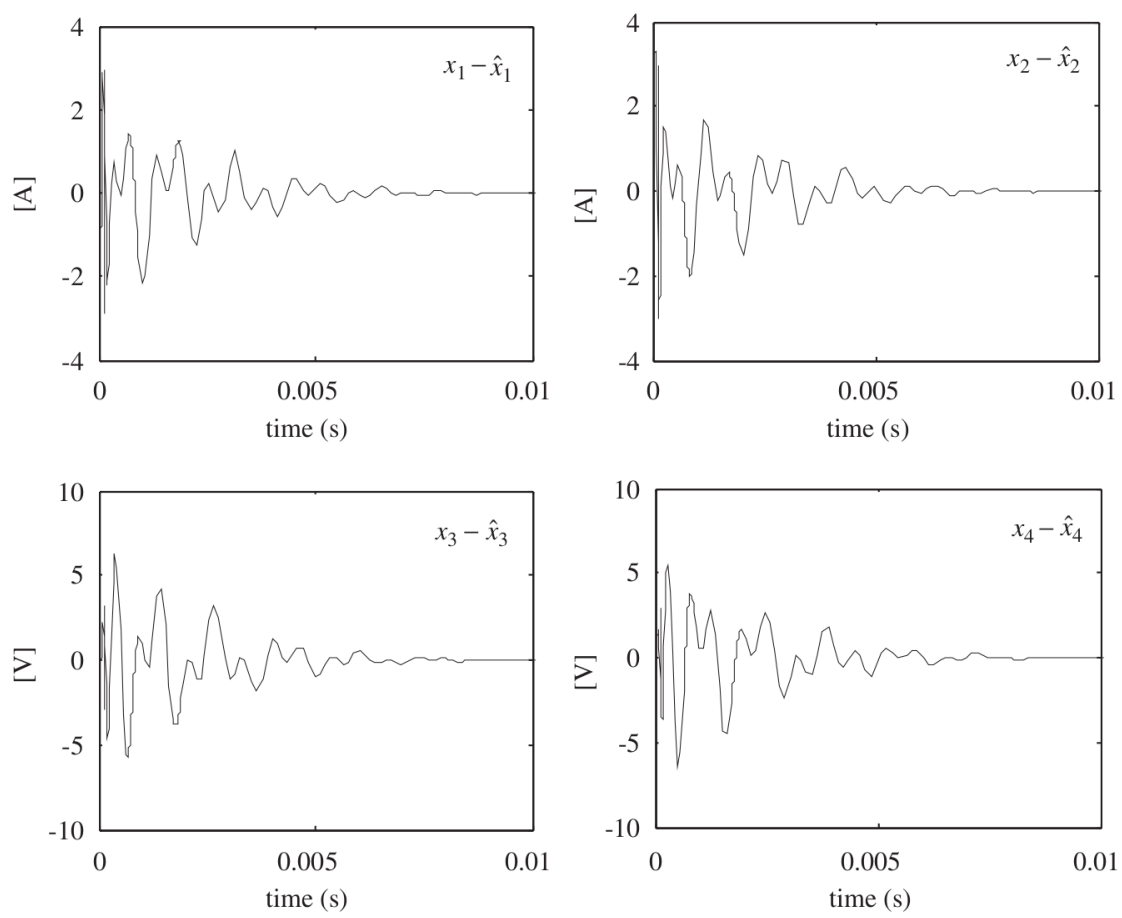

Fig. 8. State estimation errors over the first $10 \mathrm{~ms}$ transient period.
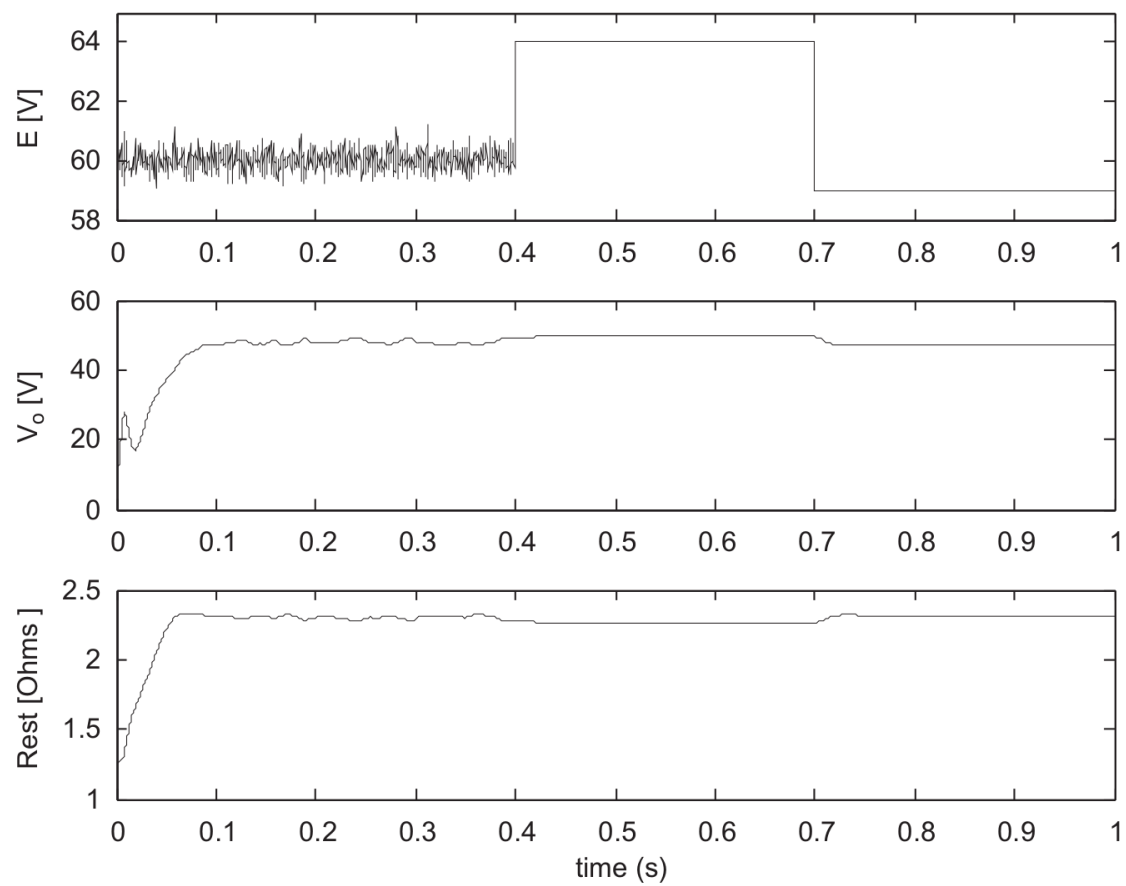

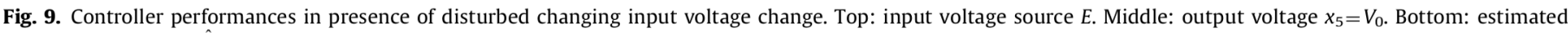
resistance load $R_{e s t}=\hat{R}$.

\subsection{Comparison with more conventional controller}

Now, the performances of the proposed nonlinear controller are compared with those of a traditional linear PI controller (Carasco et al., 2000). To make a fair comparison, both controllers are tuned to obtain a stable and as a fast closed-loop response as possible, over a wide load variation range (Fig. 11). Accordingly, the parameters of the PI controller are given the values $k_{p}=70$ and $k_{i}=5 \times 10^{3}$. The design parameters of the proposed nonlinear backstepping controller are given the values $\lambda=1 \times 10^{3}$, $c_{1}=4.5 \times 10^{3}, \quad c_{2}=1.4 \times 10^{2}, \quad c_{3}=1.2 \times 10^{2}, \quad \gamma^{-1}=1 \times 10^{16}$ and $M_{0}=10$. The performances of both controllers are illustrated in Fig. 11. It is seen that the backstepping response is faster and less oscillatory (i.e. more stable) than the PI controller. 

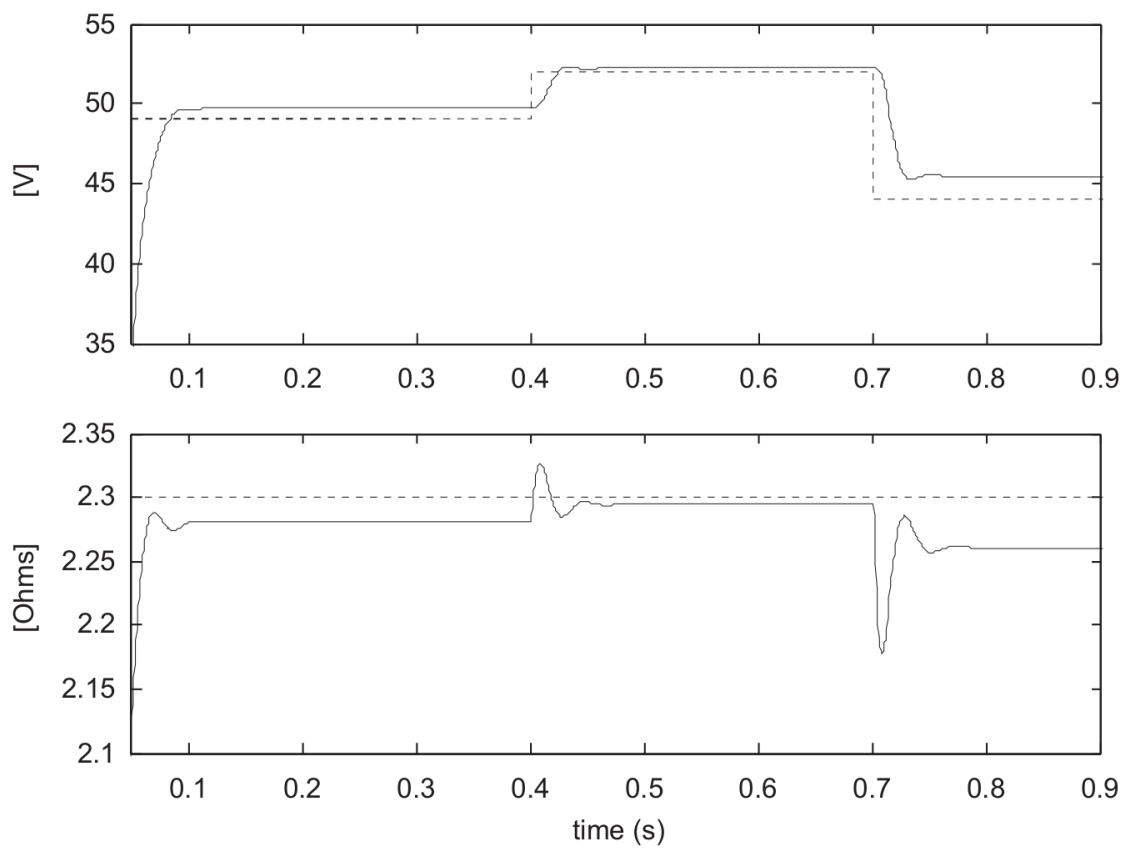

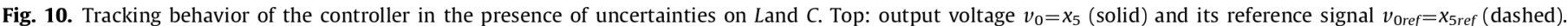
Bottom: resistance load $R$ (dashed) and its estimate $\hat{R}=R_{\text {est }}$ (solid).
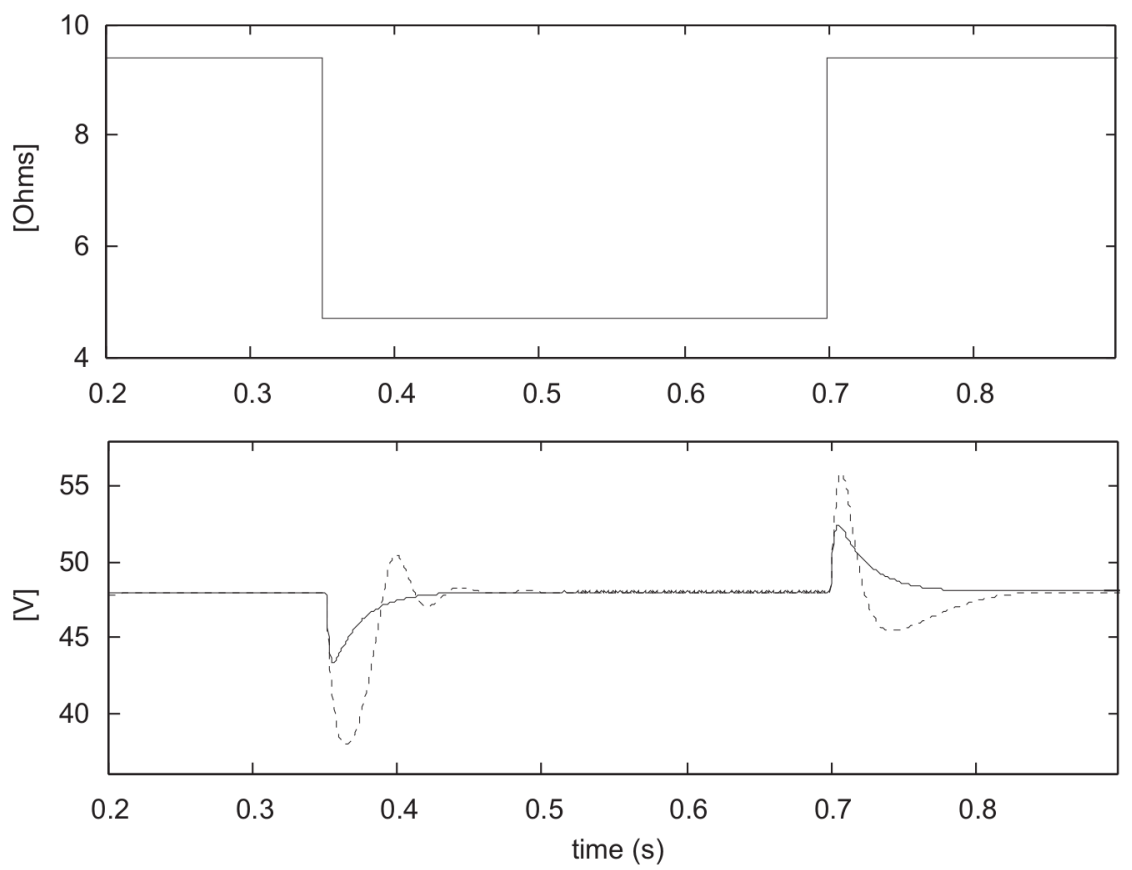

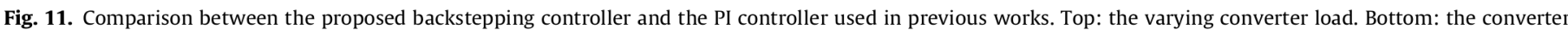
output voltage $x_{5}=v_{0}$ obtained with the proposed controller (solid) and the PI controller (dashed).

\subsection{Controller robustness against parasite load}

In this subsection, it is no longer assumed that the load is a pure resistance $R=2.3 \Omega$ (as this was the case in previous subsections). Rather, the load is let to be frequency dependent by adding to it a capacitance $C_{R}=1 \mathrm{mF}$. The proposed controller is kept unchanged, ignoring the presence of the added load capacitance. The robustness of the controller against such parasite capacitance is illustrated in Fig. 12. It is seen that the controller still ensures a tight output regulation despite the load uncertainty and parasite capacitance.

\section{Conclusion}

The problem of controlling series resonant converters has been addressed. An adaptive output feedback controller has been designed using the backstepping control technique and the high-gain observation approach. It is the first time that a controller, not necessitating the measurement of all state variables and perfect knowledge of the load, is proposed and guarantees closed-loop stability and perfect output reference tracking for this class of converters. 

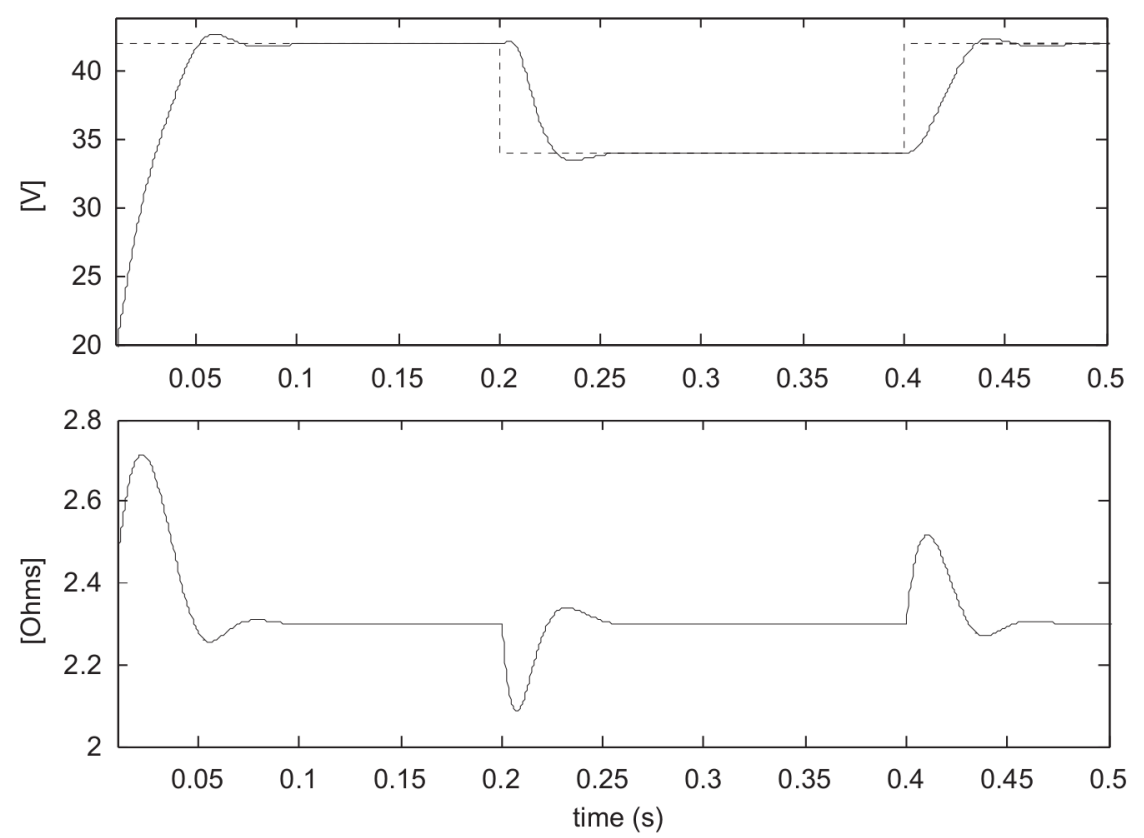

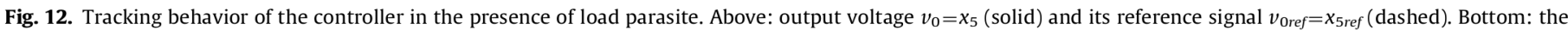
estimate resistance $\hat{R}=R_{\text {est }}$.

\section{Appendix A. Expressions of auxiliary variables}

$e_{10}=-c_{1} e_{1}+e_{2} ; \quad e_{20}=-e_{1}-c_{2} e_{2}+e_{3}-\gamma w_{1} \tau_{2}$

$x_{50}=\frac{4}{n \pi C_{0}} z_{1}+w_{1} \hat{\theta}$

$z_{10}=\frac{-\hat{z}_{3}}{L z_{1}}-\frac{2 x_{5}}{n \pi L}-\frac{2 E \hat{z}_{4}}{\pi L z_{1}}, \quad \tilde{z}_{10}=\frac{-\tilde{z}_{3}}{L z_{1}}-\frac{2 E \tilde{z}_{4}}{\pi L z_{1}}$

$a_{0}=\frac{4}{n \pi L C_{0}} \frac{\hat{z}_{3}}{z_{1}^{2}}-\frac{4 \hat{\theta}}{\pi C_{0}^{2}}, \quad a_{1}=\frac{-8}{n^{2} \pi^{2} L C_{0}}+\frac{\hat{\theta}^{2}}{C_{o}^{2}}, \quad a_{2}=\frac{4}{n \pi C_{0}^{2}} z_{1}-\frac{2 x_{5}}{C_{0}^{2}} \hat{\theta}$

\section{Appendix B. Proof of Theorem 2}

Part 1. Proof that all closed-loop signals remain bounded.

The physical variables $x_{i}(i=1, \ldots, 5)$ are bounded by Assumption A3 and (11). The state variables $z_{i}(i=1, \ldots, 7)$ are bounded by (13b) and their estimates are so by Theorem 1 . Then, one show by a direct checking that all auxiliary signals (i.e. $\left.\mathrm{ye}_{i}, g_{i}, w_{i}, \tau_{i}, \chi_{i}, \delta_{i}, \psi_{i}, \ldots\right)$ are bounded. Then, it follows from (79a) and (79b) and (83) that $\hat{\theta}$ and $u$ are in turn bounded.

Part 2. Proof of tracking performances.

First, substituting (83) for $u$ on the right side of (78) yields

$\dot{V}_{c 3}=-c_{1} e_{1}^{2}-c_{2} e_{2}^{2}-c_{3} e_{3}^{2}+\left(\tau_{3}-\frac{\dot{\hat{\theta}}}{\gamma}\right)\left(\tilde{\theta}-\gamma e_{2} w_{1}\right)+e_{3}\left(\nu+\gamma w_{1} w_{3} e_{2}\right)$

$$
+e_{3} \chi_{2}\left(e_{1}, e_{2}, e_{3}, \tilde{z}, z\right)
$$

This can be simplified considering the two cases of (81). Indeed, when $\hat{\theta}=\gamma \tau_{3}$ Eq. (B1) simplifies to

$\dot{V}_{c 3}=-c_{1} e_{1}^{2}-c_{2} e_{2}^{2}-c_{3} e_{3}^{2}+e_{3}\left(v+\gamma w_{1} w_{3} e_{2}\right)+e_{3} \chi_{2}\left(e_{1}, e_{2}, e_{3}, \tilde{z}, z\right)$

This clearly suggests the choice (84) concerning the additional action $v$. In effect, this choice makes possible to simplify further (B1) which reduces to (when $\hat{\theta}=\gamma \tau_{3}$ )

$\dot{V}_{c 3}=-c_{1} e_{1}^{2}-c_{2} e_{2}^{2}-c_{3} e_{3}^{2}+e_{3} \chi_{2}\left(e_{1}, e_{2}, e_{3}, \tilde{z}, z\right)$
On the other hand, it follows from (81) and (84) that, when $\dot{\hat{\theta}}=0$, (B1) reduces to

$$
\begin{aligned}
\dot{V}_{c 3}= & -c_{1} e_{1}^{2}-c_{2} e_{2}^{2}-c_{3} e_{3}^{2}+\tau_{3} \tilde{\theta}-\gamma \tau_{3} e_{2} w_{1}+\gamma e_{3} w_{1} w_{3} e_{2} \\
& +e_{3} \chi_{2}\left(e_{1}, e_{2}, e_{3}, \tilde{z}, z\right) \\
= & -c_{1} e_{1}^{2}-c_{2} e_{2}^{2}-c_{3} e_{3}^{2}-\gamma e_{2} w_{1}\left(w_{2} e_{2}+w_{1} e_{1}\right)+e_{3} \chi_{2}\left(e_{1}, e_{2}, e_{3}, \tilde{z}, z\right)
\end{aligned}
$$

where the last equality follows from (46), (60a) and (60b), (73) and the fact that, in the present case, $P\left(\gamma \tau_{3}\right)=0$ (because $\dot{\hat{\theta}}=0$ ). Applying the inequality $2|a b|<a^{2}+b^{2}$ with $a=e_{1}$ and $b=e_{2}$ one gets

$-\gamma e_{2} w_{1}\left(w_{2} e_{2}+w_{1} e_{1}\right) \leq \gamma \frac{w_{1}^{2} e_{1}^{2}}{2}+\gamma\left(-w_{1} w_{2}+\frac{w_{1}^{2}}{2}\right) e_{2}^{2}$

Using (44) and (55), it follows from (B5) that, when $\dot{\hat{\theta}}=0$

$$
\begin{aligned}
\dot{V}_{c 3} \leq & -\left(c_{1}-\frac{\gamma w_{1}^{2}}{2}\right) e_{1}^{2}-e_{2}^{2}\left(c_{2}+\gamma w_{1}^{2}\left(-\frac{1}{2}-\frac{\hat{\theta}}{c_{0}}+c_{1}\right)\right) \\
& -c_{3} e_{3}^{2}+e_{3} \chi_{2}\left(e_{1}, e_{2}, e_{3}, \tilde{z}, z\right)
\end{aligned}
$$

Up to now, the design parameters $\left(c_{1}, c_{2}, c_{3}\right)$ are arbitrary. Let the first one be chosen such that $c_{1}-\left(0.5+\hat{\theta} / C_{0}\right)>0$ and $c_{1}-0.5 \gamma w_{1 \max }^{2}>0$ where $\left\|w_{1}\right\|_{\infty} \leq w_{1 \max }<\infty \quad\left(w_{1 \max }\right.$ exists because $x_{5}$ is physically bounded). Given that, presently, $|\hat{\theta}|=M_{0}$ (because $\hat{\theta}=0$ ) the above inequalities suggest that $c_{1}$ must be chosen such that

$c_{1} \geq \max \left(\frac{1}{2}+\frac{M_{0}}{C_{0}}, \frac{\gamma w_{1 \max }^{2}}{2}\right)$

Using (B7), it follows from (B6) that, when $\dot{\hat{\theta}}=0$

$\dot{V}_{c 3} \leq-\left(c_{1}-\frac{\gamma w_{1 \max }^{2}}{2}\right) e_{1}^{2}-c_{2} e_{2}^{2}-c_{3} e_{3}^{2}+e_{3} \chi_{2}\left(e_{1}, e_{2}, e_{3}, \tilde{z}, z\right)$

Comparing (B3) (which holds when $\dot{\hat{\theta}}=\gamma \tau_{3}$ ) and (B8) (that holds when $\dot{\hat{\theta}}=0$ ), it follows that (B8) holds in all cases. Therefore, the latter will be based upon in subsequent developments. Applying again the inequality $2|a b|<a^{2}+b^{2}$ but this time with 
$a=e_{3}$ and $b=\chi_{2}$ one gets

$\dot{V}_{c 3} \leq-\left(c_{1}-\frac{\gamma w_{1 \max }^{2}}{2}\right) e_{1}^{2}-c_{2} e_{2}^{2}-\left(c_{3}-1\right) e_{3}^{2}+\left(\chi_{2}\left(e_{1}, e_{2}, e_{3}, \tilde{z}, z\right)\right)^{2}$

Now, recall that all physical variables (and particularly $x_{5}$ ) are bounded (Assumption A3), all estimated states $\hat{z}_{i}$ are bounded (by Theorem 1) and the parameter estimate $\hat{\theta}$ is bounded (thanks to the projection (81)). Then, using (44), (55), (57) and (A3), it follows from Eq. (76) that the state estimation errors come in linearly in $\chi_{2}\left(e_{1}, e_{2}, e_{3}, \tilde{z}, z\right)$. Then, this can be bounded from above as follows:

$\left(\chi_{2}\left(e_{1}, e_{2}, e_{3}, \tilde{z}, z\right)\right)^{2} \leq \beta V_{o b}$

for some finite real number $\beta>0$ (dependent on the signal bounds and the two parameters $\left(c_{1}, c_{2}\right)$. Using (39), one obtains from (B10)

$\left(\chi_{2}\left(e_{i: 1 \ldots 3}, \tilde{z}, z\right)\right)^{2} \leq \beta V_{o b}(0) \exp (-(\lambda-l) t)$

Combining (B9) and (B11) yields

$\dot{V}_{c 3} \leq-\alpha\|e\|^{2}+\beta V_{o b}(0) \exp (-(\lambda-l) t)$

where $\|e\|^{2}=\sum_{i=1}^{3} e_{i}^{2}$ is the Euclidean norm of the error vector $e=\left[\begin{array}{lll}e_{1} & e_{2} & e_{3}\end{array}\right]^{T}$ and

$\alpha=\min \left\{c_{1}-\frac{\gamma w_{1 \max }^{2}}{2}, c_{2}, c_{3}-1\right\}$

Integrating both sides of (B12) over $[0 \infty)$ and rearranging terms implies

$$
\begin{aligned}
& \int_{0}^{+\infty}\|e\|^{2} d t \leq V_{c 3}(0)-V_{c 3}(\infty)+\frac{\beta V_{o b}(0)}{\lambda-l} \\
& \quad \leq V_{c 3}(0)+\frac{\beta V_{o b}(0)}{\lambda-l}
\end{aligned}
$$

where the last inequality is obtained using the fact that $V_{c 3}(\infty) \geq 0$. Inequality (B14) proves that $e \in L_{2}$. On the other hand, it has already noticed that all closed-loop signals are bounded. This particularly applies to all terms on the right side of (85). Consequently, the time derivative of the error vector is in turn bounded. Since $e \in L_{2}$ and $\dot{e} \in L_{\infty}$ it follows from the Barbalat's lemma that $e$ vanishes asymptotically (e.g. Khalil, 2003). This completes the proof of Theorem 2.

\section{References}

Atassi, A. N., \& Khalil, H. K. (2000). Separation results for the stabilization of nonlinear systems using different high-gain designs. Systems $\mathcal{E}$ Control Letters, 39, 183-191.

Ahrens, J. H., \& Khalil, H. K. (2009). High-gain observers in the presence of measurement noise: a switched-gain approach. Automatica, 45, 936-943.

Besançon, G (2007). Nonlinear observers and applications. Berlin, Heidelberg: Springer.

Carasco, J. M., Escobar, G., \& Ortega, R. (2000). Analysis and experimentation of nonlinear dissipative controller for the series resonant converter. IEEE Transactions Power Electronics, 15, 536-544.

Erickson, \& Maksimivic (2001). Fundamentals of power electronics. Kluwer Academic Publishers.

El Magri, M., Giri, F., Abouloifa, A., \& Chaoui, F. Z. (2010). Robust control of synchronous motor through AC/DC/AC converters. Control Engineering Practice, 18(5), 540-553.

El Fadil, H., Giri, F., El Maguiri, O., \& Chaoui, F. Z. (2009). Control of DC-DC power converters in the presence of coil magnetic saturation. Control Engineering Practice, 17(7), 849-862.

El Fadil, H., \& Giri, F. (2009). Robust nonlinear adaptive control of multiphase synchronous buck power converters. Control Engineering Practice, 17(11), 1245-1254.

Giri, F., Liu, F., El Fadil, H., \& Elmaguiri, O. (2009). Observation of state variables in resonant DC-DC converter using the high gain design approach. In Proceedings of the IFAC symposium on power plants and power systems control (PPEPSC), Tampere, Finland.

Ioannou, P., \& Fidan, B. (2006). Adaptive control tutorial. Philadelphia: SIAM.

Krstic, M., Kanellakopoulos, I., \& Kokotovic, P. V. (1995). Nonlinear and adaptive control design. John Wiley \& Sons.

Khalil, H. (2003). Nonlinear systems. Prentice Hall.

Kazimierczuk, M. K., \& Czarkowski, D. (2011). Resonant Power Converters. John Wiley \& Sons.

Oruganti, R., Young, J. J., \& Lee, F. C. (2002). Implementation of optimal trajectory control of series resonant converters. IEEE Transactions on Industrial Electronics, 49, 318-327.

Rashid, M.H (2001). Power electronics handbook. Academic Press.

Sun, J., \& Grotstohen, H., (1993). Averaged modeling and analysis of resonant converter. In Proceedings of the IEEE Power Electronics Specialists Conference (PESC) (pp. 707-713). Seattle, USA.

Sira-Ramirez, H., \& Silva-Ortega, R., (2002). On the control of the resonant converters: a hybrid flatness approach. In Proceedings of the 15th International Symposium on Mathematical Theory of Networks and Systems. South Bend, Indiana, USA.

Sosa, A., Castilla, J. L., De Vicuna, M., Miret, L. G., \& Cruz, J. (2005). Sliding mode control for the fixed-frequency series resonant converter with asymmetrical clamped-mode modulation. IEEE International Symposium on Industrial Electronics (ISIE), 2, 675-680.

Vorperian, V. (1989). Approximate small-signal analysis of the series and parallel resonant converters. IEEE Transactions on Power Electronics, 4, 15-24.

Wong, S. C., \& Brown, A. D. (1995). Analysis, modeling, and simulation of seriesparallel resonant converters. IEEE Transactions on Power Electronics, 10, 605-614. 